\title{
DISCORSI DI COMPARAZIONE COSTITUZIONALE SUL TEMPO
}

\author{
TALKING ABOUT CONSTITUTIONAL TIME \\ IN A COMPARATIVE PERSPECTIVE
}

Anna Silvia BRUNO

RESUMEN: En este artículo, la autora se propone analizar la relación compleja entre un texto constitucional y el contexto social en el que se aplican los considerandos de los jueces como intermediarios del diálogo. La primera parte analiza la relación tiempo-sociedad en cuyos hechos se verifica la oportunidad de entender la temporalidad del sujeto inserto en aquella sociedad; ésta, a su vez, se coloca en el contexto más amplio de una situación de temporalidad natural. El segundo punto está dedicado a la relación tiempoConstitución. En la dimensión temporal, sin embargo, la Constitución cambia en diversos sentidos, algunas veces conserva el texto pero los conceptos reciben nuevas lecturas; en otras, el texto constitucional es revisado de acuerdo con el procedimiento formal pero conservando el núcleo fundamental; finalmente, suelen modificar su contenido en una perspectiva de satisfacer los intereses de una clase política o de un grupo social. El último punto está dedicado al tiempo de los jueces con particular referencia a los instrumentos utilizados en las decisiones como herramienta temporal de la actividad juzgadora.

Palabras clave: dimensión temporal; tiempo constitucional; contexto social; revisión constitucional; minimalismo judicial.
ABSTRACT: In this article, the author wants to analyze the problematic relationship between the constitutional text and the social context in which it is applied considering the judges as intermediary subjects of the dialogue. The first part is dedicated to the relationship between "time and society" in which the social facts give the opportunity to understand the individual temporality together with that of the society. The second part looks at the relationship "time-Constitution", where the Constitution can modify itself in different ways: sometimes the text remains unaltered but concepts have a new reading; other times, the constitutional text is reviewed according with the formal procedures, leaving the fundamental nucleus unchanged; finally, changing the constitutional content, its substantive aspect, in order to satisfy the interests of a political party or a social group. The last point is dedicated to the relationship between "time and judges", referring to those judicial tools which can be useful to articulate the decision making process.

Descriptors: Temporal Dimension; Constitutional Time; Social Context; Constitutional Review; Judicial Minimalism. 


\section{ARCHITETTURA TEMPORALE}

Ripensare la Costituzione non come un dato o come un fatto, bensì come un atto che continuamente si rinnova, comporta, evidentemente, l'impossibilità di prescindere dalla temporalità. ${ }^{1}$ Non più la Costituzione come realtà data situata nel tempo ma come coincidente con la temporalità stessa. In questo senso, è possibile parlare di "soggetto costituzionale"2 all'interno del circolo ermeneutico insopprimibile "tempo della Costituzione e Costituzione nel tempo" del quale sono parte attiva i giudici nell'attribuzione di un senso ai fatti che si verificano nel contesto sociale.

Nel corso di una intervista, ${ }^{3}$ il famoso architetto Daniel Libeskind ha sottolineato l'esigenza, nel progettare una costruzione,${ }^{4}$ di muovere dalla memoria, dal passato per re-iscriverlo e re-interpretarlo al fine di tutelare l'armonia dell'opera con il contesto, rispettandone la storia, la cultura ed aprendolo al trascorrere del tempo, verso una prospettiva nuova. Il contesto assume di conseguenza un aspetto diverso da quello precedente perché determinato da una scansione e da una compenetrazione di elementi temporali appartenenti a tempi diversi; tali elementi mantengono così una loro "identità" senza possibilità di essere confusi o dimenticati.

Allo stesso modo, il ripensamento del passato può essere colto come apertura o come trinceramento nel contesto sociale e giuridico. La problematicità del rapporto è data dalla ricerca di una armonia tra il tempo della Costituzione e quello della società in considerazione del fatto che i giudici, interpretando la Costituzione, intervengono come soggetti mediatori in un dialogo che necessita di essere aperto per essere democratico. "L'interpretazione della Costituzione come processo pubblico diventa il principale veicolo dell'innovazione delle Costituzioni". 5

La Costituzione fissata in un testo scritto e la società scandita dai ritmi del pluralismo culturale sono gli attori principali di un rapporto problematico in cui il giudice avverte l'esigenza di aprire il testo costituzionale

1 Rubenfeld, J., Freedom and Time, New Haven, Yale Univ. Press, 2001.

2 Per riprendere il saggio di Rosenfeld, M., L'identità del soggetto costituzionale, Lecce-Cavallino, Pensa, 2004.

3 www.cafeletterario.it/interviste/Libeskind_daniel.html

4 Nel caso specifico, il riferimento era al nuovo progetto del World Trade Center (Ground Zero) a New York.

5 Luther, J., "La Scienza häberliana delle Costituzioni", Analisi e diritto, 2001, pp. 105-143. 
al contesto in cui esso si colloca accettando i periodi di crisi della comunità: "the objective must be to write a Constitution capable of being adapted to the various crises of human affairs". ${ }^{6}$

L'articolo si struttura intorno a tre paragrafi principali che rappresentano tre differenti punti di vista: quello della società, quello della Costituzione e quello dei giudici. Nel dialogo tra i tre soggetti, il tempo emerge come prodotto della società, in termini di concezione e costruzione sociale, dal momento che esso dipende dagli strumenti con cui è calcolato per finalità meramente pragmatiche, nonché da come esso è percepito dal soggetto. Può verificarsi che la dimensione temporale dell'individuo si scontri con quella della società e, dunque, con la scansione temporale delle regole e delle istituzioni: una frattura dovuta al fatto che l'esistenza umana è in continuo movimento, che essa cresce modificandosi pur conservando una sua identità storica. In questo senso, il tempo individuale e quello sociale interagiscono nel presente che assurge a scenario in cui il passato rivive attraverso il ricordo ed il futuro è anticipato come possibilità.

Nell'aprirsi al contesto sociale di riferimento, la Costituzione è inevitabilmente soggetto temporale il cui testo scritto da un lato ne garantisce stabilità e certezza, dall'altro la espone all'attività di lettura e di interpretazione. Nella dimensione temporale, infatti, la Costituzione è destinata a mutare, talvolta lasciando invariato il testo di cui se ne intendono diversamente alcuni concetti; altre volte intervenendo revisionandolo nel rispetto delle procedure e di un nucleo fondamentale sottratto alla temporalità; altre volte ancora, modificando il suo contenuto in un'ottica di soddisfacimento degli interessi di una classe politica o di un gruppo sociale. In tutte le operazioni descritte, il potere politico si mostra determinante sia in termini di forze e strategie di partito, che in termini di potere costituente nei confronti di una Costituzione che lotta per affermare e tutelare la sua identità.

La storicità della Costituzione si coglie nel suo farsi prassi per esigenze pratiche sebbene non si esaurisca in essa: il dialogo ideale è quello tra

6 Marshall, J., M'Culloch v. Maryland, in U.S. n. 17, 316, 1819. "C'è crisi storica quando il cambiamento di mondo che si produce consiste nel fatto che al mondo o sistema delle convinzioni della generazione anteriore succede uno stato vitale in cui l'uomo si trova senza quelle convinzioni...": così J. Ortega y Gasset, Schema delle crisi, tr. it., Milano, Bompiani, 1946, p. 26. 
i suoi elementi immutabili, eterni e la struttura che ne consente il funzionamento, l'applicazione concreta immersa nella temporalità. In questo modo, è possibile cogliere un tempo che è oggettivo e soggettivo insieme. Il tempo oggettivo, quello spazializzato (chronos) che si struttura, si snoda sulla base di "istanti" numerabili, uguali tra loro anche se separati e diversi gli uni dagli altri si presenta come l'immagine complementare ed eterna della durata (aion). Il tempo soggettivo è quello individuale, della coscienza che sfugge ad ogni forma di misurazione: "il momento 'cronologico' ed il momento 'aionico' non sono dunque né autentici né esclusivi...essi danno luogo piuttosto ad una coappartenenza entro un modello unico". ${ }^{7}$

L'ultimo punto dell'articolo è dedicato al tempo dei giudici con particolare riferimento agli strumenti utilizzati nelle decisioni, simbolo di una scansione temporale dell'attività giudicante. A tale scopo, il precedente, l'applicazione diretta della Costituzione, il diritto vivente possono rappresentare gli indici rivelatori di un atteggiamento che si àncora al passato o che si fa portatore di nuove interpretazioni che si concretizzano sempre in una decisione, in una scelta di interessi da tutelare. Viceversa, nel caso dei c.d. accordi non completamente teorizzati, alla base della tecnica decisoria del Judicial Minimalism, il giudice decide di non decidere, di lasciare le decisioni "aperte" al fine di non dare soluzioni su questioni che in futuro potrebbero essere riconsiderate secondo altri canoni interpretativi.

Il fulcro del presente articolo è l'uso del tempo rispetto alla condizione dell'individuo ed al contesto sociale di riferimento; alla Costituzione in quanto testo scritto garante di stabilità e, insieme, oggetto continuo di interpretazioni e revisioni; ai giudici ed agli strumenti utilizzati nel dialogo tra i fatti e le norme.

\section{SociETÀ E COSTITUZIONE NELLA DIMENSIONE TEMPORALE}

Il fondamento del rapporto tra il soggetto e la temporalità si coglie nell'attualità e singolarità dell'esistenza umana per cui l'individuo non si esaurisce mai in un'unica situazione. Dal momento che egli si colloca in un contesto sociale che ha recepito la nozione di tempo, concretizzan-

7 Marramao, G., Kairos - Apologia del tempo debito, Bari, Laterza, 1992, p. 12. 
dola nell'uso di strumenti fisici (si pensi all'orologio o al calendario), ${ }^{8}$ è in grado di catalogare determinati avvenimenti secondo una scansione temporale. Il tempo, dunque, nasce come costruzione sociale, come simbolo attraverso cui è possibile stabilire relazioni tra eventi e dare un contenuto a quel continuum di dati che riconosciamo come passati, presenti e futuri.

La scoperta da parte dell'uomo di "esserci", di collocarsi cioè in un determinato ambiente caratterizzato da molteplici possibilità, lo spinge ad oltrepassare l'azione presente ponendosi di volta in volta come "poter-essere" verso altre possibilità. ${ }^{9}$ All'interno della struttura sociale, il soggetto non si pone come spettatore di una realtà immutabile ma si rivela nella sua capacità di progettare, utilizzando le cose come strumenti per formare e trasformare il mondo e se stesso. Infatti, la "produzione di possibilità di tipo nuovo", la "selezione delle possibilità utilizzabili" con esclusione di quelle inutilizzabili e la "stabilizzazione delle possibilità utilizzabili"10 rappresentano le funzioni principali di cui ogni sistema necessita per potersi evolvere. Nel tempo, l'uomo

elabora la sua visione del mondo traverso riferimenti continui, traverso scoperte incessanti, traverso rettifiche e modificazioni, aggiunte e rimaneggia-

8 L'orologio come strumento attraverso cui "la modalità di coglimento incarnata nella fisica si fa vivente, e con essa si fa vivente il modo in cui il tempo ha l'occasione di mostrarsi" è in M. Heidegger, Il concetto di tempo, tr. it., Gallio, Ferrara, 1990, 17. L'interdipendenza tra individuo, società e natura, per cui i mutamenti storico-sociali del primo sono strettamente connessi a quelli degli altri due soggetti, è alla base del discorso sul tempo di Elias, N., Saggio sul tempo, tr. it., il Mulino, Bologna, 1986, pp. 59 ss. e 103 e ss.

9 Così secondo l'impostazione di Heidegger, M., Tempo ed essere, Napoli, Guida Editori, 1969, pp. 132 e ss. Il pensiero di Heidegger sulla temporalità è stato determinante per Paul Ricoeur che ha trattato il problema dell'unità del tempo in Tempo e racconto, Milano, Jaca Book, 1988, vol. III, pp. 99 e ss. Su Heidegger, M. e Geymonat, L., Storia del pensiero filosofico e scientifico, Milano, Garzanti, 1975, pp. 177 e ss. L'esistenza basata sull'idea heideggeriana di essere in vista di è indicativa delle molteplici possibilità che l'uomo scopre nel mondo senza esaurirsi in nessuna di esse. L'esistenza dell'uomo nel mondo è poter-essere, apertura alle cose ed agli altri. Il prendersi cura delle cose e l'aver cura degli altri costituiscono i momenti fondativi di una esistenza inautentica (deiezione, ossia la caduta dell'uomo sul piano delle cose, dei fatti) o di una esistenza autentica, secondo coscienza: Reale, G. e Antiseri, D., Il pensiero occidentale dalle origini ad oggi, Brescia, Ed. La Scuola, 1983, vol. 3, p. 449.

10 Luhmann, N., La differenziazione del diritto, il Mulino, Bologna, 1990, p. 39. 
menti portati quotidianamente alla propria immagine della realtà, al proprio concetto del mondo, alla propria idea delle cose. ${ }^{11}$

Nel lavoro di costruzione, di architettura, il passato è recuperato e ritematizzato grazie alla memoria, mentre il futuro è progettato per la capacità dell'uomo di elaborarlo. Per potersi sviluppare, la memoria soggettiva necessita, a sua volta, di elementi istituzionali (quali il linguaggio, la scrittura, le commemorazioni ecc.) che sono il prodotto di un processo storico e che consentono di non dimenticare ciò che è accaduto, rivisitandolo più volte. Attraverso la memoria storica, l'uomo ricostruisce la propria identità riprendendo un passato che non scompare ma che è negli oggetti, nei sentimenti, nei ricordi.

Se la memoria aiuta l'uomo a non dimenticare, l'oblio lo spinge ad aprirsi al nuovo perché non rimanga chiuso in un passato che può ritornare nella storia sotto forma di violenza, di guerre, creando fratture incolmabili tra i popoli ed eliminando ogni possibilità pacifica di dialogo (così per la frattura insanabile tra irlandesi/inglesi; ebrei/palestinesi; ecc.). Nel presente rivivono il passato attraverso il ricordo ed il futuro attraverso l'attesa e la progettazione: come in una fusione di orizzonti, il futuro appartiene al passato e si realizza come possibilità, come aspettativa. "Passato", "presente" e "futuro" sono concetti che racchiudono non solo l'essere un "prima", un "ora" e un "dopo", ma anche "gli uomini che attuano la sintesi e vedono davanti a sé il corso degli avvenimenti e la sua struttura temporale... cos'è passato, presente e futuro dipende dalle generazioni che vivono in quel momento". ${ }^{12}$ I concetti elaborati in un determinato periodo storico sono soggetti a nuove possibilità interpretative perché variano con il variare della concezione che ha l'uomo di essi, considerando che tali concezioni si presentano mutevoli in quanto l'esperienza umana che le ha prodotte è mutevole. ${ }^{13}$

Ogni società tende a costruire una propria dimensione temporale ed una propria organizzazione sociale del tempo per percepire passato, presente e futuro. Nella società occidentale contemporanea, in particolare,

11 Capograssi, G., Analisi dell'esperienza comune, Milano, Giuffrè, 1975, pp. 35 e ss., in cui l'Autore sottolinea la necessaria interazione tra l'uomo che si affaccia al mondo e che ad esso si apre ed il mondo che lo accoglie.

12 Elias, N., op. cit., nota 8, pp. 93 e ss.

13 La differenza tra concetto e concezione è di Dworkin, R., L'impero del diritto, Milano, Il Saggiatore, 1989, 71. 
le possibilità offerte si moltiplicano rapidamente, i tempi si accorciano e le distanze si coprono a velocità senza precedenti: l'uomo vive un processo di continue trasformazioni causate dalle innovazioni tecnologiche legate alla comunicazione, al trasporto, alla produzione. Le nuove forme di organizzazione economica, gli attuali modelli occupazionali, le costanti riorganizzazioni e "razionalizzazioni" strutturali, la computerizzazione - solo per fare pochi esempi- favoriscono una accelerazione dei tempi sociali ed economici ${ }^{14}$ determinando il corso della storia. Sempre più spesso ormai, significativi cambiamenti si sviluppano all'interno della medesima generazione piuttosto che a conclusione o ad apertura di un corso generazionale. Le grandi organizzazioni e istituzioni sociali sembrano regolare il tempo, quel tempo scandito dall'orologio, indicativo di un tempo spazializzato, oggettivo perché misurabile, quantificabile; un tempo, cioè, contrapposto a quello soggettivo, della coscienza. ${ }^{15}$

Nella dimensione temporale, il rapporto individuo/società non è mai statico poiché si struttura intorno e per mezzo di due soggetti che non sono né "isolati", né "immobili". Alla ricerca di una necessaria interazione, l'uomo si affaccia al mondo e si apre ad esso; il mondo lo accoglie e si manifesta con "la ricchezza delle sue determinazioni, tutto il sistema delle sue posizioni, tutto il patrimonio sempre attivo delle sue operazioni". ${ }^{16}$ Pertanto, il singolo è colto nel suo essere nel mondo, nel suo essere un prodotto del proprio, singolare tempo che si intreccia con il tempo della società in cui egli opera.

14 Così evidenziato da Scheuerman, W. E., Constitutionalism in age of speed, in Constitutional Commentary, vol. 19, n. 2, 2002, 359-360, e da Kay, R. S., "Constitutional Chronomy", Ratio Juris, vol. 13, n. 1, 2000, pp. 34 e ss.

15 Sul ruolo della coscienza, si veda Elias, ivi, per cui: "la coscienza da cui parte l'osservazione degli oggetti è essa stessa un oggetto: é un oggetto che diventa soggetto nel corso di due processi, quello sociale - pratico di civilizzazione; l'altro, individuale culturale di coscientizzazione", per cui l'uomo è in costante cambiamento, è esso stesso un processo. Attraverso la coscienza ogni evento si manifesta sul piano relazionale e su quello causale rispetto ad altri eventi, passati e futuri, frutto di un atto intimo che nel flusso della durata mantengono una loro specificità che li distingue. Ogni momento conserva i momenti precedenti ed allo stesso tempo è nuovo rispetto ad essi. La coscienza, dunque, vive il presente nella intertestualità con il passato ed il futuro; è l'avanzare del passato che incombe sull'avvenire, alimentandosi continuamente nel suo procedere, ed è perciò un continuo crescere su se stessa. Coscienza come memoria, conservazione del passato nel presente ma anche anticipazione dell'avvenire.

16 Capograssi, G., op. cit., nota 11, pp. 35 e ss. 
Gli scopi che una società intende perseguire diventano parte integrante di un "flusso continuo di eventi" in cui si inserisce quel continuum di fatti creato dal singolo. Da qui il pluralismo sociale e giuridico delle società attuali, per cui, istanze e fatti sempre nuovi rinviano a valori costituzionalmente tutelati in attesa di una loro concretizzazione, mentre, la certezza del diritto è continuamente minata, mai completamente realizzata. Sebbene al diritto sia richiesto di garantire la sicurezza giuridica, esso non si sottrae alla durata che si presenta come evoluzione creatrice del "nuovo" e che determina un'armonia sociale fondata sull' equilibrio tra la stabilità ed il mutamento. Si può allora parlare di evoluzione continua o di trasformazione controllata laddove la funzione del diritto non si risolva esclusivamente nell'analisi dell'equilibrio del sistema ma tenga conto, invece, delle turbolenze, delle discontinuità, degli stati di transizione. È il tempo che potremmo definire "di metamorfosi", ${ }^{17}$ fondato sul cambiamento graduale di un organismo la cui identità rimane inalterata.

Guardando alle trasformazioni della società, la Costituzione ne coglie la sua struttura pluralistica, le sue situazioni di multiculturalità e la ricerca di nuovi codici di convivenza da parte di culture differenziate. Il pluralismo sociale caratterizzato dalla nascita di nuovi gruppi e movimenti, da forme di cooperazione e dissenso è sintomo di un tempo storico che non è più quello di una società-monade, isolata e chiusa, bensì il tempo di processi storici nazionali e internazionali. Sul piano giuridico, dall'apertura della Costituzione al contesto sociale derivano problemi legati alla interpretazione della Costituzione, alla revisione costituzionale ed alle politiche costituenti quali piani di operatività degli elementi di conservazione, innovazione e pluralismo culturale. ${ }^{18}$ Il rapporto tra processi/interpretazioni e atti/leggi costituzionali non può, allora, che considerarsi in fieri, dal momento che è recepito di volta in volta all'interno di un flusso continuo di eventi, tale per cui esso si coglie come prodotto di un contesto spazio-temporale di una particolare società. ${ }^{19}$ Le dimensioni temporali convivono in un tempo presente in cui si elaborano pregiudizi

17 Così Ost, F., Le temps du droit, Paris, O. Jacob, 1999, p. 248.

18 La concezione häberliana della Costituzione come cultura è ripresa da Luther, J., op. cit., nota 5, pp. 105-143.

19 Cassirer, E., "Il problema dello spazio e del tempo nella metafisica e nella teologia", Storia della filosofia moderna, vol. II, tr. it., Torino, Einaudi, 1953, p. 485; sul profilo della dimensione spaziale in cui collocare il rapporto fatti-norme, mi si permetta il rinvio a Bruno, A., "Il diritto vivente nello spazio postmoderno", in Tondi della Mura, 
e si realizzano valori che appartengono alla storia di un popolo, di cui ne hanno determinato l'identità. Questi valori e questi principi recuperati dal passato attraverso nuove interpretazioni consentono di creare continuità storica proiettandosi verso il futuro.

I valori presenti nelle Costituzioni e nelle Dichiarazioni universali, dei quali si fanno portatori i principi fondamentali, richiedono continue attuazioni ${ }^{20}$ e necessarie aperture al contesto culturale in cui sono inseriti. Nella ricerca della regola più adeguata alla soluzione del caso, il giudice deve sia considerare l'ambiente di riferimento, sia valutare la possibilità che subentrino riforme legislative, considerando le eventuali ricostruzioni interpretative dell' ordinamento vigente. Queste operazioni risultano evidenti laddove si tratti di un caso che si impone come nuovo rispetto alla tradizione e che si qualifica come nuovo nel momento in cui si prende coscienza della sua novità attraverso l'analisi di senso e di valori cui esso rinvia. ${ }^{21} \mathrm{Il}$ processo di studio del singolo caso deve necessariamente essere contestualizzato nella dimensione temporale in considerazione degli avvenimenti sociali che lo hanno prodotto, cioè degli elementi in grado di indicare un caso come "critico", "difficile":22 guardando al cambiamento delle circostanze, infatti, la regola giuridica è resa oggetto di una interpretazione adeguata alla trasformazione dell'ordinamento complessivamente inteso. La Costituzione ha bisogno, da un lato, di guardare ai mutamenti sociali, al sorgere di nuovi conflitti, all'esigenza di soluzioni e di interpretazioni sempre nuove, alla richiesta da parte delle istituzioni di regole astratte e generali — nella ricerca di una residua certezza del e nel diritto-; dall'altro, necessita di valutare le modalità, le possibilità con-

V. et al. (cur.), Corte Costituzionale e processi di decisione politica, Torino, Giappichelli, 2005, pp. 439 e ss.

20 Interessante la distinzione tra i concetti di applicazione, esecuzione ed attuazione, laddove quest'ultima risulta essere in grado di dare applicazione ai principi impliciti, svincolandosi dal testo, Ainis, M., "Attuazione di norme a mezzo di norme", Giur. Cost., 1996, n. 3, 2021.

21 Il rapporto senso-significato inquadrato in una struttura circolare rende la comunicazione un fenomeno circolare che «non "contiene" altro che se stessa e che perciò necessita di essere analizzata in un "lungo periodo", in un'ottica di "sviluppo storico"»: così Cerroni, U., "Formale e informale", Scienzasocietà, settembre-dicembre 1986, p. 7.

22 Si parla di casi critici nel momento in cui c'è disaccordo sul senso da attribuire al fatto in questione, per cui, diversi "intendimenti" e "comprensioni" comportano diverse “soluzioni giuridiche”, Zagrebelsky, G., Il diritto mite, Torino, Einaudi, 1992, p. 191. 
crete dei principi di realizzarsi nella soluzione delle controversie e nella concretizzazione di quei valori dei quali essi si fanno portatori.

I principi fondamentali sono lo strumento di cui si avvale la Costituzione nella soluzione delle controversie, considerando che essi

per essere effettivamente tali e, dunque... effettivi (ponendosi a base di un ordine sociale e giuridico che sempre rimane fedele alla sua matrice originaria, pur rinnovandosi senza sosta), devono rivelarsi testimoni di un presente che non rinnega la propria storia ovvero, rovesciando, di una storia che si prolunga senza fratture nel presente: essere e divenire a un tempo, anzi essere nel divenire. Perciò, essi assolvono ad una funzione che è conservativa e promozionale insieme, mantenendo vivi i valori originari, di cui costituiscono la (pur imperfetta) traduzione in termini positivi, ed ulteriormente alimentandoli e proiettandoli verso ancora maggiori sviluppi, offrendo, "copertura" e garanzia agli atti (ed ai fatti) che vi danno specifica attuazione avverso palesi violazioni o subdoli tentativi di regresso rispetto alle conquiste oggimai fatte. I principi, insomma, ricostruiscono attorno a se medesimi, al loro nucleo espressivo e qualificante, una trama assiologico-normativa in costante tensione ed evoluzione. ${ }^{23}$

\section{LA COSTITUZIONE NEL TEMPO}

L'habitus individuale si costruisce sulle basi di quello sociale e si sviluppa attraverso il linguaggio quale strumento sociale di comunicazione $^{24}$ che permette la comprensione ed il riconoscimento tra i soggetti. Il linguaggio trasmette la nostra storia, la nostra appartenenza culturale e l'individuo si relaziona con la società, con l'altro. ${ }^{25}$

23 Ruggeri, A., "L'identità costituzionale alla prova: i principi fondamentali fra revisioni costituzionali polisemiche e interpretazioni-applicazioni «ragionevoli»", Ars Interpretandi, 1996, n. 1, p. 117.

24 Elias, N., op. cit., nota 8, pp. 26-27; e 167-168.

25 Sulla crisi del rapporto con l'Altro e con gli altri nei confronti dei quali l'individuo scopre l'esistenza di una "discrepanza integrale", Ortega y Gasset, J., op .cit., nota 6, pp. 173 ss. "O tempo da transormação é implacavelmente no presente, è sempre simultâneo às premissas e às decisões, independentemente se «por si» ou «por outros»... A experiência constitucional è tudo isto: è contingência do processo decisório": così Carducci, M., Por um Direito Constitucional Altruísta, Livraria do Advogado, 2003, pp. 68 e ss. Sul rapporto tra diritti umani e democrazia in un tempo di "crise funcional" e "conceitual", Bolzan De Morais, J. L., As Crises do Estado e da Constituição e a Transformação Espacial dos Direitos Humanos, Porto Alegre, Livraria do Advogado, 2002, pp. 18 e ss. 
Il linguaggio, inteso come insieme segnico cui attribuire un senso, sviluppa il discorso sia sul piano sincronico, per cui un fatto è semplicemente un termine con più significati, che su quello diacronico, di comprensione e non di sola spiegazione. In questa direzione, esso opera in quanto strumento principale con cui poter dare vita ad un procedimento di riforma di un testo e, dunque, anche di quello costituzionale. ${ }^{26}$

\section{La Costituzione come testo}

Con il termine "testo" si intende un discorso di qualsiasi genere fissato dalla e nella scrittura e nella sua funzione esso si completa nel rapporto con la lettura che rende possibile la sua apertura. Con la scrittura il "mondo" dell'autore si riversa nel testo mentre il lettore lo interpreta attraverso spiegazione e comprensione, non solo e non tanto per cogliere l'intenzione dell'autore quanto per confrontarsi con il testo e percepirne il senso. Con la scrittura, per un verso l'autore comunica il suo pensiero; per altro, se ne libera e lascia che il testo si apra all'esterno: il lettore ne percepisce la struttura, le relazioni interne ma, soprattutto, si appresta alla sua comprensione.

Con riferimento al testo costituzionale, la norma contenuta in un dispositivo richiede, oltre ad un'attività di comprensione, anche quella di pre-supposizione e di pre-giudizio. ${ }^{27}$ Infatti, nella interpretazione del testo, l'obiettivo principale dell'interprete è quello di rimanere ad esso legato pur allontanandosene per ragioni pratiche ma riconducendo ad esso gli esiti del suo allontanamento.

La Costituzione è un testo le cui relazioni interne sono continuamente rielaborate dall'interprete nelle attività di "politica costituzionale", ${ }^{28}$ che

26 Se però il linguaggio è un insieme segnico, non ogni segno è un simbolo: quest'ultimo, infatti, richiede la natura polisemica del termine che, in quanto tale, aziona un processo ermeneutico basato sulla plurivocità e non sul rapporto significante-significato. A. Rigobello, Prefazione a Ricoeur, P., Tempo e racconto, Milano, Jaca Book, 1986, vol. I, p. 8: "Il segno è simbolo, rimando, provocazione interpretativa: in prima lettura va letto dal punto di vista dell'archeologia del soggetto, ossia nella prospettiva del passato, nella serie delle determinazioni che legano il segno alla sfera dell'inconscio e ai suoi dinamismi; in seconda lettura si scorge nel segno la «sovradeterminazione» del simbolo, il suo significato si chiarisce nella finalità volta al futuro".

27 Gadamer, H. J., op. cit., nota 85, p. 316.

28 Zagrebelsky, G., op. cit., nota 22, pp. 150 e ss. 
permettono di ridefinire non solo i significati delle disposizioni costituzionali ma anche il senso stesso della Costituzione (attività di configurazione e ri-figurazione). All'interno di un testo, mediante le operazioni narrative che operano sul linguaggio, si formano e si intrecciano le azioni ed i soggetti (configurazione) per cui emerge l'esperienza viva del tempo che non si esaurisce nella pura fenomenologia ma rinvia alla "coscienza storica della esistenza", alla continua reinterpretazione in considerazione del contesto di riferimento (ri-figurazione). ${ }^{29}$ In questo modo, attraverso l'esperienza temporale il contenuto del testo assume un significato perché la sua narrazione è la ri-figurazione della esperienza temporale del lettore, cioè della temporalizzazione della esperienza. ${ }^{30}$

Nella rilettura e riscrittura del testo costituzionale, la dimensione temporale si concretizza in un'attività legislativa che opera erga omnes mentre l'intervento sul suo fondamento ha efficacia meramente persuasiva. Quest'ultimo è il risultato di un'attività interpretativa, frutto di un "incontro tra una oggettività da interpretare (testo) ed una soggettività interpretante, entrambe immerse in un contesto intersoggettivo di significati". ${ }^{31}$ È evidente il ruolo fondamentale dello strumento con cui si esprime la Costituzione, ossia il linguaggio, intendendo "tanto la struttura linguistica" con la quale i principi sono rappresentati, "quanto la

29 Ricoeur, P., Tempo e racconto, cit., nota 26, vol. III, pp. 124 e ss.

30 Si veda, Elias, N., Il processo di civilizzazione, tr. it., Il Mulino, Bologna, 1988, 96 ss. Nel rapporto individuo-società emerge, da un lato, la individuazione del soggetto e dall'altro la socialità dell'azione. Teoria dell'azione e teoria del sistema con rinvii al pensiero di N. Luhmann sono alla base del discorso intorno al rapporto individuo-società di F. Pardi, Osservazioni sul concetto di osservazione, pp. 39-40 in Strazzeri, M. (cur.), La sintesi possibile. Saggi su Norbert Elias, Lecce, Pensa Multimedia, 2000. Per Elias, N., "Il concetto di «figurazione» è un semplice strumento concettuale col cui aiuto ci possiamo liberare dalle costrizioni sociali a parlar e pensare come se «individuo» e «società» fossero due figure diverse ed inoltre antagoniste... Con figurazione noi intendiamo il modello mutevole (costituito dai giocatori intesi come una totalità...E' evidente che questa figurazione costituisce una struttura di tensione, perché l'interdipendenza dei giocatori, presupposto del loro formare insieme una figurazione specifica, può configurarsi come una interdipendenza tra alleati oppure tra avversari". Cfr. Strazzeri, M., Introduzione a Strazzeri M. (cur.), op. cit., pp. 92 e ss.

31 Modugno, F., Interpretazione costituzionale e interpretazione per valori, in www. costituzionalismo.it, 08/07/2005. 
struttura linguistica di cui sono dotati gli atti (costituzionali e non) che vi apprestano alla prima". ${ }^{32}$

Qualora volessimo analizzare alcuni fatti rappresentativi di processi temporali utilizzando le figure proprie del processo espressivo, potremmo considerare la famiglia di fatto e quella fondata sul matrimonio. Nella sentenza della Corte costituzionale n. 166/98, il ragionamento del giudice considera la tutela della filiazione uno degli elementi in comune ai due nuclei. Grazie all'uso della metafora potremmo dire che essa

contribuisce alla produzione del significato, rafforzando la relazione tra le similarità in riferimento ad un codice o, in altre parole, collegando i segni lungo un asse paradigmatico... La metafora conduce verso un livello astratto in cui ciascun individuo è uguale agli altri, in quanto portatore di una necessità di relazioni intime ed in quanto ha diritto di perseguire liberamente l'appagamento di tale bisogno fondamentale. ${ }^{33}$

La metafora risulta appropriata perché scaturisce da una rapporto analogico di base che permette di cogliere le somiglianze tra cose differenti ma congeneri. Allo stesso tempo, però, operare sul piano metaforico vuol dire individuare una serie di caratteristiche uguali che sollevano evidentemente problemi legati al contesto culturale in senso ampio. ${ }^{34} \mathrm{Da}$ tale processo, infatti, potrebbero emergere delle "identità incompatibili con l'ordine costituzionale", ${ }^{35}$ con un codice comune che consente un dialogo continuo sullo stesso territorio tra gruppi sociali, etnici, religiosi differenti e che rende possibili difficili bilanciamenti a tutela degli interessi emersi.

Sebbene la metafora abbia un fondamentale "potenziale creativo", risulta ugualmente necessario che essa venga collocata in un sistema, in una logica strutturale che aiuti sia ad esprimere tale potenziale, sia a "dis-

32 Ruggeri, A., op. cit., nota 23, p. 118.

33 Rosenfeld, M., op. cit., nota 2, pp. 40 e 42.

34 "Si innesca perciò un rapporto di costante, mutua alimentazione tra la dimensione etica e culturale (naturalmente, in senso antropologico) del vivere associato ed il piano giuridico-costituzionale, nel cui ambito le grandi opzioni assiologiche si sono tradotte e -in qualche modo-fissate, quasi cristallizzate", D'Andrea, L., Ragionevolezza e legittimazione del sistema, Milano, Giuffrè, 2005, pp. 220-221.

35 Rosenfeld, M., op. cit., nota 2, p. 39. 
ciplinarlo (limitandolo e definendolo)", ${ }^{36}$ attraverso rapporti tra varianti di senso e classi di contesto. Da qui, l'esigenza di affrontare il discorso anche delle differenze (ancora sentt. n. 166/98 e sent. n. 233/94 della Corte costituzionale), ${ }^{37}$ individuando quelle che contraddistinguono due situazioni che tra loro potrebbero sembrare simili, risolvibili ricorrendo all'analogia ma che in realtà necessitano di una differenziazione in un'ottica di bilanciamento di interessi. Così, il tema della filiazione nelle famiglie di fatto è affrontato considerando non solo e non tanto le somiglianze tra le due situazioni - in un'ottica di salvaguardia degli interessi della prole, ritenuto interesse rilevante- quanto, in primo luogo, prendendo atto delle differenze fondamentali ${ }^{38}$ tali per cui:

36 Grampa, G., "Introduzione”, in Ricoeur, P., La metafora viva, Milano, Jaca Book, 1981, pp. XI e ss.

37 Entrambe su www.giurcost.it.

38 Sent. n. 166/98: "Il valore costituzionale di tutela della filiazione trova concreta specificazione nelle disposizioni previste dagli artt. 147 e 148 del codice civile, che, in quanto complessivamente richiamate dal successivo art. 261, devono essere riguardate nel loro contenuto effettivo, indipendentemente dalla menzione legislativa della qualità di coniuge, trattandosi dei medesimi doveri imposti ai genitori che abbiano compiuto il riconoscimento dei figli naturali. Il primo obbligo enunciato dall'art. 147 del codice civile consiste in quello di mantenimento della prole: é questo un dovere inderogabile, che nella sua concreta attuazione é commisurato in proporzione alle rispettive sostanze dei genitori e alle capacità di lavoro di ciascuno. Procede per necessità da ciò che i provvedimenti giudiziali inerenti all'entità dell'obbligo, poiché questa é rapportata ad elementi variabili nel tempo, sono soggetti a modifica in conseguenza del mutamento della situazione di fatto. L'assolutezza dell'obbligo in esame e l'indissolubilità del suo legame con il rapporto di filiazione sono confermati dall'intervento imposto dal legislatore agli altri ascendenti legittimi o naturali, che sono tenuti, quando i genitori siano privi di mezzi sufficienti, a fornire ai genitori stessi i mezzi necessari affinché possano adempiere al loro dovere di cura nei confronti dei figli, il quale dovere resta inderogabilmente a carico dei genitori... La tutela dell'interesse della prole rappresenta infatti la ratio in forza della quale il legislatore, prevedendo la disciplina circa l'assegnazione della casa familiare in sede di separazione dei coniugi, ha introdotto il criterio preferenziale, ancorché non assoluto, indicato dal quarto comma dell'art. 155 del codice civile, la cui applicazione é rimessa alla valutazione del giudice in relazione alla situazione concreta. Sotto questo profilo l'obbligo di mantenimento si sostanzia quindi nell'assicurare ai figli l'idoneità della dimora, intesa quale luogo di formazione e sviluppo della personalità psico-fisica dei medesimi; onde l'attuazione di detto dovere non può in alcun modo essere condizionata dalla assenza del vincolo coniugale tra i genitori, poiché la fonte dell'obbligo de quo agitur é unica, ma sufficiente: quella del rapporto di filiazione. La mancanza di una specifica norma che regoli le conseguenze, riguardo ai figli, della cessazione del 
Il giudice rimettente premette anzitutto di non aderire a quell'orientamento giurisprudenziale e dottrinario, che reputa applicabile per analogia alla famiglia di fatto la disciplina normativa della famiglia legittima ed in particolare il provvedimento di assegnazione della casa familiare di cui all'art. 155, quarto comma, del codice civile, in quanto la emanazione di tale provvedimento si fonda sul necessario presupposto del matrimonio, come, del resto, si desume dal tenore letterale della disposizione in oggetto, che fa riferimento al "coniuge cui vengono affidati i figli", non già al "genitore"... Ed in effetti, attesa la collocazione della norma nel capo relativo allo scioglimento del matrimonio e alla separazione dei coniugi, correttamente è escluso il ricorso all'analogia, in quanto essa presuppone la similarità delle situazioni, la quale, oltre a non essere presente tra il rapporto coniugale e quello di mera convivenza in sé considerati, non é voluta dalle parti, che nel preferire un rapporto di fatto hanno dimostrato di non voler assumere i diritti e i doveri nascenti dal matrimonio; onde la imposizione di norme, applicate in via analogica, a coloro che non hanno voluto assumere i diritti e i doveri inerenti al rapporto coniugale si potrebbe tradurre in una inammissibile violazione della libertà di scelta tra matrimonio e forme di convivenza. ${ }^{39}$

rapporto di convivenza di fatto dei genitori non impedisce allora di trarre da una interpretazione sistematica delle norme in tema di filiazione la regula iuris da applicare in concreto, senza necessità di ricorrere all'analogia, né ad una declaratoria di incostituzionalità. L'interprete é infatti al cospetto di un sistema perfettamente coerente con i principi costituzionali, nel quale é già contenuta la norma che gli consente di regolamentare, ex latere filii, le conseguenze della cessazione della convivenza di fatto: la linea di guida cui egli deve attenersi é l'interesse del figlio alla abitazione, come al mantenimento, correlato alla posizione di dovere facente capo al genitore. L'assegnazione della casa familiare nell'ipotesi di cessazione di un rapporto di convivenza more uxorio, allorché vi siano figli minori o maggiorenni non economicamente autosufficienti, deve quindi regolarsi mediante l'applicazione del principio di responsabilità genitoriale, il quale postula che sia data tempestiva ed efficace soddisfazione alle esigenze di mantenimento del figlio, a prescindere dalla qualificazione dello status (sentenza n. 99 del 1997)".

39 Sent. n. 233/94: "Nell'ottica di tale disposizione costituzionale è stato pure affermato dal questa Corte che la tutela di dette minoranze può richiedere la predisposizione di un trattamento specificamente differenziato (sentenza n. 86 del 1975), in forza del principio di «eguaglianza sostanziale» e della connessa esigenza di forme di tutela positiva. É stato, in proposito, riconosciuto di recente (sentenza n. 438 del 1993) che alle minoranze (nella specie, di lingua tedesca e ladina) è costituzionalmente garantito anche il diritto di esprimere in condizioni di effettiva parità la propria rappresentanza politica. Va infine rilevata la tendenza, di cui è espressione la recente legge costituzionale 23 novembre 1993, n. 2, ad estendere l'ambito di tutela delle minoranze linguistiche anche oltre i gruppi minoritari fino ad oggi considerati. Ma è altrettanto evidente che tale tutela non può superare certi limiti, dovuti ad una serie di diverse considerazioni (anche di propor- 
Con la metafora l'accento si pone sul rapporto tra "l'evento ed il sistema", "sulla tensione tra termine singolo e frase", in quanto, affrontare tale discorso in termini esclusivamente di strutture e capacità logiche diventa riduttivo essendo esse analoghe in tutte le circostanze. ${ }^{40}$ Infatti, lo strutturalismo tende a rielaborare i miti quali "sistemi" validi in tutti i tempi e, dunque, in una prospettiva in cui non c'è tempo ma solo un "eterno presente" - dimensione verso cui si tende utilizzando il precedente per uniformare decisioni differenti-. Attraverso la metafora vi$\mathrm{va}^{41} \mathrm{o}$ attraverso il rinvio al binomio metafora-metonimia ${ }^{42}$ il linguaggio diventa creativo e veritativo consentendo di ri-figurare la realtà, di creare un senso nuovo, di ri-descrivere il passato attualizzandolo in un presente che non è più considerato come dato, ma come possibilità, come poter essere. ${ }^{43}$ Quest'ultima fase identifica l'uomo nel suo essere-nel-mondo —riprendendo Heidegger — poiché "il momento del «comprendere» risponde dialetticamente all'essere in una situazione come progetto dei possibili più propri entro le situazioni stesse in cui ci troviamo". ${ }^{44}$ Ugualmente, il ruolo dell'interprete si concretizza nella scelta dei "significati" intesi come potenzialità linguistiche. ${ }^{45}$

\section{Revisione e nucleo essenziale della Costituzione}

La scrittura e la lettura della Costituzione rendono possibile la conoscenza della storia di uno Stato, dei suoi principi fondamentali quali con-

zionalità numerica) e soprattutto al necessario contemperamento di questa esigenza con altri valori parimenti meritevoli di tutela".

40 Ricoeur, P., "La contestazione dello «strutturalismo»", in Tempo e racconto, cit., nota 26 , pp. 260 e ss.

41 Ricoeur, P., op. cit., nota 36.

42 "Il legislatore è vincolato dal principio per cui un trattamento diseguale è giustificato quando le differenze di fatto sono tali dal giustificare la differenziazione", Lucarelli, A., "L'effettività del principio di eguaglianza e diritti sociali nei processi evolutivi della forma di stato", Il Filangieri, Gennaio-Marzo, 2004, p. 93.

43 Sul concetto di "intenzione" determinato da risposte alla domanda "perché", ossia da risposte che escludono un spiegazione attraverso la causa, Ricoeur, P., La semantica dell'azione, Milano, Jaca Book, 1986, pp. 64 e ss., e 95.

44 Heidegger, M., Il concetto, cit., nota 9, p. 27.

45 Dogliani, M., "Diritto costituzionale e scrittura", Ars Interpretandi, n. 2, 1997, p. 134. 
quista di conflitti politici e giuridici, della struttura e del funzionamento del testo, degli organi cui compete la sua applicazione. In particolare, il tema della revisione della Costituzione è un punto centrale dell'analisi del testo e del suo rapporto con la temporalità. Da un lato, esso è collegato alla questione di legittimità e di efficacia dell'ordinamento poiché investe il procedimento di produzione del diritto positivo; dall'altro, al riconoscimento di autorità del potere costituente su cui si basa la legittimità della Costituzione. ${ }^{46}$

Parlare di revisione della Costituzione vuol dire chiedersi se esiste o meno un complesso di principi e regole che ne compongono un "codice genetico" a-temporale, rappresentativo di un limite strutturale alle modifiche del testo costituzionale. Si tratta di un nucleo composto da "clausole di eternità" teso a salvaguardare l'integrità essenziale del sistema costituzionale, ${ }^{47}$ le esigenze di stabilità, certezza e coerenza ${ }^{48}$ ma che convive insieme ad una serie di variabili destinate, invece, a modificarsi nel tempo, adattandosi alle esigenze del contesto culturale. In altre parole, il fondamento della Costituzione, il suo impianto portante è stato raffigurato ${ }^{49}$ non solo come necessario, essenziale ed indivisibile, determinante per la tutela dei diritti fondamentali e per il riconoscimento della identità della Costituzione, ma anche dinamico. Esso, infatti, pur garantendo la sua integrità e la sua persistenza nella evoluzione temporale, non sottrae la Costituzione alla esigenza di eventuali cambiamenti, espressione del potere costituente, al contrario, allontana i rischi di frantumazione della struttura. Pertanto, stabilendo il percorso attraverso cui modificare la Costituzione, il processo di revisione costituzionale sarebbe in grado di garantire una relativa stabilità delle regole costituzionali evitando la fragilità politica del testo (a condizione che il potere politico non violi le regole previste per la procedura di revisione). Nel rispetto della identità della Costituzione - simbolo della storia, della cultura, dei conflitti che hanno segnato un Paese, un popolo ed il sistema di riferimento, nonché

46 Longo, G., "Il concetto di mutamento costituzionale in Hans Kelsen e Carl Schmitt", Rivista Internazionale di Filosofia del Diritto, 1996, LXXIII, p. 259.

47 Camerlengo, Q., Contributo ad una teoria del Diritto costituzionale cosmopolitico, Milano, Giuffrè, 2007.

48 Carducci, M., Tecniche Costituzionali argomentazione, normazione, comparazione, Lecce, Pensa Multimedia, 2003, pp. 77 e ss.

49 Camerlengo, op. cit., nota 47. 
le procedure giuridiche su cui esso poggia - $\mathrm{i}$ valori ed i principi in essa contenuti costituiscono il "nucleo duro", 50 un "limite" alla revisione costituzionale.

La revisione del testo costituzionale interviene nello scenario sociopolitico come un atto che rompe l'armonia, la stabilità di un ordinamento che si era riconosciuto, fino a quel momento, in un determinato dettato costituzionale. L'attività di revisione può essere ritenuta, per un verso, meramente giuridica, per cui, l'intero testo costituzionale è disancorato dal trascorrere del tempo (dai mutamenti giuridici, sociali, politici,...) ed assoggettato esclusivamente al potere sovrano previsto nella Costituzione originaria.

Queste Leggi Fondamentali, irremovibili, difficili da cambiare, devono dirigere la vita dello Stato grazie al loro potere irrefrenabile. Non possono essere modificate dai poteri stabiliti, possono solo essere modificate secondo la propria previsione, di non facile applicazione. Queste Leggi Fondamentali dirigono la vita statale, in virtù della loro forza che persiste nel tempo. ${ }^{51}$

D'altra parte, l'attività di revisione può essere intesa come politicamente legittimata dal potere dominante e costitutivo di un momento storico in cui si avverte la minaccia alla stabilità dell'ordinamento. Pertanto, il senso attribuito alla Costituzione ed i valori che da essa emergeranno non saranno più quelli sostenuti dai partiti politici dominanti nel momento costituente ma quelli che si affermeranno nel corso delle pratiche costituzionali.

Sul piano della revisione costituzionale, i procedimenti servono ad integrare le generazioni future nell'arco costituzionale, adeguando la Costituzione a trasformazioni culturali sopravvenute o pilotando tali trasformazioni in tempi ragionevoli. Anche i fallimenti delle revisioni devono essere analizzati sotto il profilo delle loro determinanti culturali, compito di particolare attualità in

50 Idem e Ruggeri, A., op. cit., nota 23, p. 114: "A conti fatti, cosa siano i principi, come percepirne l'essenza e fino a che punto essi resistano al mutamento costituzionale, rimangono (e, forse, sempre rimarranno...) questioni oggetto di accese, non ripianate controversie... I valori dello Stato di diritto e dei diritti dell'uomo ha indotto taluni a parlare di un diritto costituzionale comune trans-statale che...sarebbe un complesso di principi giuridici che potrebbero servire da fondamento a norme costituzionali nazionali": Troper, M., Il futuro del diritto costituzionale, Lecce-Cavallino, Pensa, 2004, p. 21.

51 Jellinek, G., Mutamento e riforma costituzionale, Lecce, Pensa, 2004, p. 17. 
Italia. La funzione innovativa prevale ma non deve sacrificare la conservazione dell'identità di una Costituzione, individuabile mediante "clausole pro aeternitate" e mediante il riferimento agli elementi del modello dello stato costituzionale democratico. ${ }^{52}$

Nel primo caso, la revisione costituzionale rischia di ridursi a "mera finzione", priva di legittimità politica e disancorata da quei processi che consentono alla Costituzione di non esaurirsi in un documento "formale". Nel secondo caso, il potere costituente - la cui dimensione spaziotemporale è sempre circoscritta - legittimando l'attività di revisione acquista continuità storica. In questo modo, è garantita una certa stabilità alla "legittimità democratica del sistema costituzionale" 53 senza recidere quella idea di continuità che si era realizzata fino a quel momento.

Un aspetto particolare del tema della revisione costituzionale è offerto dalla lettura di una Costituzione che guarda se stessa nel cambiamento: il riferimento è alla ipotesi specifica dell'autoemendamento. ${ }^{54}$ In questo caso, l'idea di forza della Costituzione sarebbe interna ad essa e supportata da forze ed indirizzi politici che mirano a fare della Costituzione un testo capace di contestualizzarsi, di legittimarsi attraverso una norma che gli attribuisce "significanza sociologica". Tale procedimento di autoreferenzialità è possibile se sostenuto da scelte politiche che lo legittimano. Infatti, la ratio sottesa alla clausola dell' autoemendamento sembra essere quella di evidenziare come la Costituzione sia un soggetto temporalizzato per cui essa "non può rappresentare un totem intangibile, pena la sostituzione traumatica o un esteso ricorso all'ambiguo sistema degli aggiustamenti in via di prassi e delle modifiche non espresse, che comunque oltre certi limiti erodono le garanzie e la legittimità dell'ordinamento"), ${ }^{55}$

52 Luther, J., op. cit., nota 5., pp. 105-143.

53 De Vega García, P., "Mondializzazione e diritto costituzionale: la crisi del principio democratico nel costituzionalismo attuale", Diritto pubblico, 2001, n. 3, p. 1086.

54 Suber, P., The paradox of Self-Amendment, New York, Peter Lang, 1990, pp. 197 e ss. Sul puzzle dei diritto costituzionale di A. Ross, R. Guastini, Lezioni di teoria analitica del diritto, Torino, Giappichelli, 1982, pp. 72 e ss., e Longo, G., op. cit., nota 46, p. 261.

55 Rizza, G., "Autoreferenzialità, decostruzione, cambiamento nella revisione costituzionale: spunti critici e riflessioni”, Diritto e società, 2002, n. 1, 30, per cui, l'adattamento della Costituzione alla "evoluzione della società e al dibattito politico... nel rispetto della continuità e della legalità costituzionale" rappresenta "una soluzione che consente anche di tutelare il diritto delle generazioni future di influire a loro volta 
nonché quella di contenere e gestire gli eventuali mutamenti delle circostanze che richiedono, in qualche modo, una reinterpretazione del testo o un suo adeguamento alla realtà passando per il processo di revisione costituzionale. ${ }^{56}$ Naturalmente, questo comporta una valutazione in termini di armonia costituzionale, cioè in termini di non-contrasto tra il testo revisionato ed il testo costituzionale ed una valutazione delle conseguenze, dei rischi che una riforma può generare e del consenso che sostiene la riforma stessa.

\section{A. Ipotesi di diacronia temporale}

La necessità da parte del testo costituzionale di aprirsi alla "evoluzione" della società si rivela necessaria al fine di rintracciare nuovi assetti culturali attraverso "indicatori di prevedibilità" forniti dal dialogo e dalla negoziazione tra soggetti e gruppi di soggetti che partecipano ai processi decisionali. ${ }^{57} \mathrm{Si}$ realizza in questo modo l'adattamento di un testo al suo contesto di riferimento, costruendo la base di ciò che è stato definito

sui capi saldi dell'ordinamento che le governa". "La dottrina dell'integrazione (personale, materiale e funzionale) rifiuta la concezione tradizionale dello Stato come entità immobile, statica, costruita a partire dai tre «elementi» territorio, popolo e potere statale, proprio perché questa concezione impedisce di comprendere lo Stato come realtà vitale": Smend, R., Costituzione e diritto costituzionale, Milano, Giuffrè, 1988, p. 287.

56 Come esempio, si pensi alla riforma del Titolo V della Costituzione, per cui " attraverso un legittimo procedimento di revisione costituzionale, che avrebbe dovuto incidere soltanto sulla parte organizzativo-funzionale dei rapporti tra Stato, Regioni ed Enti locali, occorre ripensare l'effettività del principio di eguaglianza sostanziale, quale paradigma fondativo dei diritti sociali e della democrazia", Lucarelli, A., op. cit., nota 42, p. 93. Bilancia, F., "La riforma del Titolo V della Costituzione e la «perdurante assenza di una trasformazione delle istituzioni parlamentari»", Giur. Cost., 2004, fasc. 1, pp. 139 e ss., in cui si sottolinea la necessità di ricorrere a strumenti giurisprudenziali al fine di rendere flessibile il nuovo testo normativo a tutela degli interessi e dei valori ad esso sottesi. La democrazia, "como vir-a-ser cotidiano precisa estabelecer vínculos fortes como a vida; é um estado de compromisso daqueles que participam deste processo...": Bolzan De Morais, J. L., A subjetividade do tempo, Porto Alegre, Livraria do Advogado, 1998, p. 106.

57 Hsü Dau-Lin, Mutación de la Constitución, Bilbao, IVAP, 1998, p. 169. Carducci, M., Tecniche Costituzionali, cit., nota 48, p. 92. M. D'Amico riprende P. Haberle, Zeit und Verfassunng (1974) in Riflessioni sulla nozione di tempo nel diritto costituzionale, in Jus - Rivista di Scienze giuridiche, anno XXXIX, 1993, n. 1, 73: "porre l'accento sulla concretezza del tempo costituzionale significa riconoscere l'importanza di questo pro- 
"tempo storico" e che sarebbe il "tempo della Costituzione" dal momento che ne determina i «contenuti e le finalità». ${ }^{58}$ Il tempo "nella Costituzione", invece, si può distinguere in tempo astratto e tempo concreto. Il tempo astratto è un tempo eterno, in cui l'astrattezza diventa l'elemento capace di comprendere innumerevoli fattispecie senza esaurirsi in esse; quello concreto è un tempo oggettivo, misurabile, come, ad esempio, quello proprio degli organi costituzionali nello svolgimento delle loro funzioni, nell'esercizio degli adempimenti normativi. ${ }^{59}$

La Costituzione intesa come soggetto è, dunque, soggetto storico appartenente ad un tempo storico in quanto ad esso si piega nelle sue manifestazioni, nelle sue espressioni di concretezza (nei fatti) rimanendo immutabile e garantendo stabilità. Essa si colloca nella storia, è essa stessa storia — "non è «temporale» perché «sta nella storia», ma, al contrario, esiste e può esistere storicamente soltanto perché è temporale nel fondamento del suo essere"-; ${ }^{60}$ è tempo storico che perdura imponendosi come identità narrativa capace di mutare senza frantumarsi:

sotto l'aspetto dell'innovazione, la Costituzione è pertanto anche "l'espressione di una situazione di sviluppo culturale" (kultureller Entwicklungszustand). La cultura dà vita e forma a quella Costituzione più o meno buona in cui un popolo "è" la sua Costituzione materiale o il contesto che determina l'efficacia e lo sviluppo della Costituzione formale (il testo). L'immagine häberliana della "Costituzione come processo culturale" sviluppa l'idea smendiana della Costituzione come processo dinamico d'integrazione il cui motore sono più le pratiche comunicative e simboliche della negoziazione e della tolleranza reciproca che non le armi. Questo processo culturale si rinnova da una generazione all'altra attraverso le cognizioni e le emozioni, attraverso procedimenti di rielaborazione del passato e di proiezione di speranze sul futuro. La cultura della Costituzione dipende non soltanto dai progressi della "ingegneria" di politologi e giuristi, ma è innanzitutto determinata dalla cultura generale della cittadinanza che la accetta e dal radicamento dei suoi principi nell'etica civica. ${ }^{61}$

cesso, di questo continuo mutamento, come un aspetto unico della dimensione temporale della Costituzione".

58 D’Amico, M., op. cit., nota 57, p. 50.

59 Ibidem, p. 71.

60 Heidegger, M., "Temporalità e storicità", in Bianco, F. (cur.), Il dibattito sullo storicismo, Bologna, Il Mulino, 1978, p. 313.

61 Luther, J., op. cit., nota 5, pp. 105-143. 
Quando si verificano avvenimenti storici che segnano passaggi importanti, momenti costituzionali fondamentali indice di una trasformazione in corso e di una "rottura" con il passato, si avverte l'esigenza di intervenire sul testo costituzionale per colmare una eventuale frattura con il suo contesto, in un'ottica di consolidamento della democrazia. Dal momento che l'assetto di una società può subire nel tempo dei mutamenti, è necessario che le stesse disposizioni costituzionali, per essere effettivamente vigenti, siano adeguate al nuovo assetto sociale. Il procedimento di revisione costituzionale costituisce uno strumento che consente di inserire la Costituzione formale nella storicità propria della Costituzione materiale e di dare continuità al potere costituente. ${ }^{62}$

Questo processo di adeguamento messo in atto dalle forze socio-politiche dovrebbe tendere a creare punti di equilibrio tra una pluralità di soggetti, forme di integrazione e di armonia tra testo e contesto, regole di disciplina con un indirizzo prestabilito. In un quadro così delineato, il ruolo chiave è svolto dalla politica che deve operare nel rispetto dei limiti espliciti ed impliciti alla revisione costituzionale (sebbene sia altrettanto possibile che anche nel rispetto delle norme relative al procedimento di revisione si possa incidere sull'ordinamento politicoistituzionale alterandone l'identità: questo accade, ad esempio, quando le modifiche coinvolgono gli elementi tipici della forma di Stato e di governo). Nel rapporto "Costituzione (come norma giuridica) e politica (come sistema delle scelte di opportunità)...la trasformazione costituzionale è intesa come «accoppiamento strutturale» di diritto e politica, come «fondamento inquieto» dell'intero sistema giuridico composto di fatti e volontà". ${ }^{63}$ In un contesto democratico, esiste un rapporto di dipendenza tra l'operatività, l'applicazione della Costituzione e la sfera della politica intesa in tutte le sue espressioni, dalla responsabilità dei parlamentari alle manifestazioni dell'opinione pubblica, al fine di individuare regole che fungono da filtri costituzionali delle scelte, dei comportamenti e che confermano la Costituzione come "legge suprema" senza "banalizzarla"

62 De Vega García, P., op. cit., nota 53, pp. 1086 e ss.

63 Carducci, P., "Presentazione", in Posada, A., Genesi della Costituzione e Consuetudine, Lecce-Cavallino, Pensa, 2004, p. 10. 
degradandola a livello di una legge ordinaria, sprovvista di strumenti che le consentano di difendersi dalle intromissioni della politica. ${ }^{64}$

Negli ultimi anni numerose sono state le riforme costituzionali, tra cui quella proposta dalla legge costituzionale n. 1/1997 relativa alla seconda parte della Costituzione; le leggi costituzionali n. 1/1999 e n. 3/2001 che hanno riformato il titolo V della parte II della Costituzione; il disegno di legge approvato in prima lettura dalla Camera e dal Senato (rispettivamente il 15 ottobre 2004 ed il 23 marzo 2005) relativo ad una ulteriore modifica della parte II della Costituzione. In queste occasioni, si è proceduto dapprima derogando all'articolo 138 con l'obiettivo di poter snellire una procedura già di per sé meno complessa di quelle previste da altre Costituzioni, successivamente approvando a maggioranza la più ampia revisione degli ultimi tempi.

Infatti, nonostante l'obiettivo fosse quello di riformare solo gli aspetti organizzativi compresi nella seconda parte della Costituzione, in realtà, sono stati toccati i contenuti "etico-politici fondamentali" 65 del testo, $\mathrm{i}$ punti funzionali al mantenimento della identità costituzionale (evidente il rinvio in seno alla riforma all'art. 5 della Costituzione ed al contenuto essenziale dei diritti), le garanzie della Costituzione (titolo VI), la Corte costituzionale ed il CSM, gli stessi limiti al potere di revisione: si pensi alla "forma repubblicana" (al carattere democratico della Repubblica e dell'appartenenza della sovranità al popolo), ai diritti "inviolabili" dell'uomo, alla Repubblica come "una ed indivisibile", alla rigidità costituzionale (la revisione del Titolo $\mathrm{V}$ ha dato vita al regionalismo differenziato facendo leva sul negoziato, sulla intesa tra Stato-Regioni (art.116, ult. comma) attenuando, nelle materie indicate, il carattere rigido della Costituzione).

La Costituzione è stata oggetto di interventi e modifiche frutto di compromessi politici talvolta in contrasto con la struttura e la storia costituzionale. In questo senso, i progetti elaborati in Italia dalle ultime due Commissioni bicamerali e quello presentato dal centro-destra nel 2005, hanno proposto l'introduzione del Senato federale, un nuovo riparto di

64 Così evidenziato da M. Volpi, La banalizzazione della Costituzione tra revisioni adottate e riforme progettate, su www.costituzionalismo.it, 13/06/2005.

65 Dogliani, M. e Massa Pinto, I., La crisi costituzionale italiana nell'attuale fase della “lotta per la Costituzione”, su www.costituzionalismo.it, 15/02/2006. 
competenze tra Stato e Regioni ed un nuovo procedimento legislativo che metterebbe fine al bicameralismo perfetto

[introducendone uno che] non si sa di che tipo ma pessimo, e con un innesto certo anche se camuffato nella composizione della Corte costituzionale che snaturerebbe il ruolo di quest'organo di garanzia, prezioso, delicatissimo, quanto mai necessario, tanto più con i tempi che corrono, per salvaguardare almeno un po' di stato di diritto, per garantire un'eguaglianza almeno formale e assicurare qualche credibilità al catalogo dei diritti costituzionalmente riconosciuti. 66

Punto fondamentale della riforma è l'attribuzione di totale supremazia al Primo Ministro (che, solo per indicare pochi punti essenziali, determinerebbe la politica nazionale, nominerebbe i Ministri senza l'intervento del Capo dello Stato, non avrebbe bisogno della fiducia delle Camere) e la conseguente riduzione dei poteri del Capo dello Stato (che non potrebbe nominare il Premier né sciogliere le Camere, né avrebbe il potere di autorizzare i disegni di legge di iniziativa del Governo) che determina tra i ruoli uno squilibrio sconosciuto a tutte le attuali democrazie; inoltre,

la possibilità di chiedere sempre il referendum costituzionale in caso di modifica della Costituzione a prescindere dalla maggioranza schieratasi a favore della modifica (per cui) la trasformazione del referendum da oppositivo in confermativo stravolge il disegno costituzionale per il quale il ricorso al popolo è l'extrema ratio di chi vuole impedire la revisione. ${ }^{67}$

La Costituzione è apparsa altrettanto "maltrattata" a seguito dell' approvazione della Legge n. 270 del 21 dicembre 2005 con cui il Parlamento ha modificato il sistema per l'elezione dei membri della Camera dei deputati e del Senato della Repubblica, definita da più parti come "irrazionale", "di comodo", di cui sono denunciate le contraddittorietà. ${ }^{68}$ La legge elettorale ha sollevato non pochi dubbi sulla sua costituzionalità (con riferimento al premio di maggioranza, al principio della uguaglianza tra i sessi, al metodo...) senza poter essere per questo oggetto di controllo di

66 Ferrara, G., Per la critica al progetto di riforma della Costituzione del governo Berlusconi, su www.costituzionalismo.it, 02/09/2003.

67 M. Dogliani e I. Massa Pinto, op. cit., nota 60.

68 Tra gli altri, Zagrebelsky, G., "La riforma irrazionale", su La Repubblica, 25-102005. 
costituzionalità prima della sua applicazione, segnando perciò un gap incolmabile tra il tempo costituzionale della politica e quello costituzionale della garanzia e del giudizio. Detto in altri termini, il sistema giuridico, sociale, politico avverte una delusione delle aspettative del singolo a vedere la realizzazione dei propri diritti. ${ }^{69}$ Una legge sottratta ad una valutazione in termini di impatto normativo e di conseguenze sociali; una legge, ancora, che mina l'identità di una Costituzione, identità di un popolo, escluso e delegittimato nel processo della sua trasformazione (si pensi al ribaltamento dei risultati conseguiti con il referendum del 1993).

La Costituzione italiana, invece, si manifesta in tutta la sua fragilità e si piega al potere contingente della politica, dei rapporti di forza, delle strategie e dei vantaggi dei partiti uscendone indebolita sul piano del riconoscimento, dell'attuazione, della concretizzazione dei principi portanti e dei diritti costituzionali. Si assiste ad un tempo che potremmo definire "diacronico"70 in cui il tempo di ciascuno di poter costruire il futuro secondo le aspettative anche in funzione del passato ${ }^{71}$ cioè dei diritti acquisiti e dei principi e valori fondamentali riconosciuti e che ne rappresentano il "nucleo essenziale" non sembra possa essere definito e talvolta realizzato perché ostacolato dal tempo della politica, in cui si affermano norme costituzionali di breve durata, prive del carattere di generalità e astrattezza.

\section{DiMENSIONE TEMPORALE NELLE DECISIONI DEL GIUDICE.}

\section{IL PRECEDENTE}

La concretizzazione dei principi costituzionali può verificarsi " $a$ opera o del legislatore per mezzo di una regola che guarda agli accadimenti

69 Sulla necessità che i diritti fondamentali vengano recepiti in norme positive, tale per cui si genera il nesso normativo tra aspettative e garanzie e la successiva questione di "realizzabilità concreta delle garanzie", si veda ancora Ferrajoli, L., Diritti fondamentali, Bari-Roma, Laterza, 2002, pp. 30 e ss.

70 Ost, F., op. cit., nota 17, p. 37.

71 Zamorano Farçias, R., Civilizzazione delle aspettative e democrazia nelle periferie della società moderna, Lecce, Pensa Multimedia, 2003, pp. 123 e ss., considerando che la società "non è altro che un fascio di aspettative stabilizzate", si distinguono aspettative cognitive, culturali che nascono dal bisogno di non restare delusi di fronte alla società; e aspettative normative che invece mantengono la loro natura di aspettativa nonostante le delusioni. 
futuri, o dal giudice per mezzo di una decisione che guarda agli accadimenti passati". ${ }^{72}$

Nell'attività giudiziale, nel momento in cui un giudice decide quale norma applicare al caso specifico, evidentemente attribuisce, prima di ogni altra cosa, un senso a quel fatto. L'attribuzione di senso, caratterizzando la struttura portante di quel fatto, produce inevitabilmente un certo grado di certezza (all'interno delle dimensioni di senso). Nell'ambito della dimensione temporale, tale attribuzione è possibile per la presenza di elementi costanti (oggetti e situazioni) e variabili (eventi) ed è caratterizzata dal rinvio ad ulteriori possibilità presentandosi come reale (attuale) e possibile (potenziale) insieme: nel momento in cui un senso si attualizza, per sua natura rinvia ad altre possibilità che rimangono in fieri.

Il giudice nel presente, "ora", può proiettarsi in una dimensione temporale futura oppure guardare al passato cercando in esso una soluzione: in quest'ultimo caso la dottrina del precedente facilita un "trinceramento" dietro soluzioni già adottate e che, per una continuità dell'ordinamento, possono essere utilizzate per disciplinare i casi futuri. Rinviando a decisioni già prese per la soluzione di un caso nuovo, il giudice può, dunque, guardare al passato come strumento di continuità ritematizzandolo in un'ottica "evolutiva", anticipando, cioè, la possibile "evoluzione" di un "senso", nel rispetto delle proprie radici culturali.

Nonostante il differente ruolo nei paesi di common law e civil law il precedente giudiziario sembra essere uno strumento in grado di garantire una certa stabilità all'interno del sistema come un "filtro" per le attribuzioni di senso a "tutela" e nel rispetto di valori tradizionali comuni. ${ }^{73}$ In particolare, all'interno del sistema di common law, dove il diritto è considerato in termini di risultato (mentre in quello di civil law è ritenuto un motore della dinamica sociale rivolto verso il futuro), il rinvio al precedente è sostanzialmente un vincolo che fa leva su una regola che si giustifica nel nome della "prevedibilità, della stabilità e della

72 Zagrebelsky, G., "Diritto per: valori, principi o regole?", Quaderni Fiorentini, 2001, n. 3, Milano, Giuffrè, p. 876. Sulla dimensione temporale quale elemento basilare dell'attività del legislatore (che si occupa di regolare il futuro) e del giudice (che rende eterna una norma applicandola a tutti quei casi che presentano somiglianze), D'Amico, op. cit., nota 57, pp. 60 e ss.

73 Rosenfeld, M., Interpretazioni, Bologna, il Mulino, 2000, pp. 41 e ss. 
fallibilità" 74 che il giudice dispone a fondamento del sistema. Dal momento che in questo discorso è evidente l'uso del "passato", è opportuno precisare che guardare al passato come precedente è cosa diversa che considerarlo una mera esperienza. ${ }^{75}$ In quest'ultima ipotesi, i fatti passati, rispetto a quelli attuali da decidere, rilevano "ora" per la loro reciproca somiglianza e dunque in quanto tali, senza che nella somiglianza emergano caratteristiche sostanziali, ma meramente fenomenologiche.

Non è così nel caso in cui si tratti di precedente, poiché esso opera alla stregua di una regola e perciò, sebbene esso possa essere ritenuto errato, comunque influenzerebbe la decisione attuale quale argomento fondato su una regola, su una "autorità". Si è sottolineato come argomentare secondo una regola comporti operare un atto di "generalizzazione" che è proprio della regola stessa, della sua natura, della sua struttura, mentre il precedente richiede volta per volta la costruzione di tale generalizzazione, con riferimento cioè ai casi che si verificano. Naturalmente, il ricorso alla dottrina del precedente si giustifica anche per una esigenza di continuità rispetto ai principi ed alle scelte del passato, attraverso un "continuum interpretativo". ${ }^{76}$ Raramente si verificano due casi perfettamente identici che consentono di risolvere quello attuale utilizzando quello passato: il vincolo imposto dal precedente opererebbe nel senso di poter "controllare" tutti i fatti ad esso successivi e che sono "come" quello passato assunto a parametro di precedente. ${ }^{77}$

74 Schauer, F., Le regole del gioco, Bologna, il Mulino, 2000, p. 280. Il precedente è preso in considerazione come elemento in grado di «contrastare determinate aspettative, ma anche di minacciare la sicurezza delle aspettative, vale a dire la loro sicura prevedibilità»: così Luhmann, N., Sistemi sociali, Bologna, il Mulino, 1990, p. 474.

75 Schauer, F., op. cit., nota 74, p. 280.

76 Ibidem, pp. 280-281. Ma anche Pugiotto, A., Sindacato di costituzionalità e "diritto vivente", Milano, Giuffrè, 1994, p. 81.

77 "...è impossibile che il diritto, che vuole coscientemente agire nel futuro, possa disporre di norme per regolare tutti gli avvenimenti futuri imprevedibili. Per questo, si desume dall'esperienza storica il fatto che ogni Costituzione presenti delle lacune che si manifestano, spesso, dopo molto tempo e non possono essere colmate mediante i mezzi convenzionali dell'interpretazione e dell'analogia": così Jellinek, G., op. cit., nota 51, p. 52. Sul diritto come integrità che richiede la valutazione, da parte del giudice, del grado di coerenza della sua interpretazione affinché intacchi "l'integrità in misura inferiore rispetto alle interpretazioni rivali", considerando che un giudice "che abbia maturato una certa esperienza conoscerà abbastanza bene il terreno nel quale affonda il suo problema più prossimo per comprendere istintivamente quale interpretazione di un insieme di casi 
Una volta riconosciuto simile, (il precedente) non mette in gioco nessun altro criterio se non quello della sua capacità di universalizzazione (trattare in modo simile i casi simili). Problematico è, però, il riconoscere la similitudine; dire che tale situazione diventa un precedente significa già operare una selezione nel thesaurus delle sentenze di giustizia. Sotto quali aspetti due casi sono simili? Si ritorna alla "valutazione" rispettiva delle somiglianze e delle differenze. Pur accordandoci su di loro, rimarrebbe il problema di sapere quali somiglianze e quali differenze sono pertinenti nella vicenda che si considera. ${ }^{78}$

Dal momento poi che le generalizzazioni devono essere costruite, tale opera di architettura è messa in atto dal giudice del secondo caso, cioè del caso che si è sviluppato in un tempo presente; analizzando le circostanze determinanti per entrambi i casi, egli (il giudice del secondo caso) potrà realizzare una struttura capace di fare astrazione dai casi particolari, di generalizzare, comprendendo entrambi gli eventi (quello passato e quello presente) oppure considerandone solo uno dei due, essendo innumerevoli le generalizzazioni ipotizzabili. In questo processo, la possibilità di ricavare una regola, una generalizzazione da un certo numero di casi è data dal fenomeno della "sotto-determinazione" tale per cui si creerebbero dei sotto-insiemi formati dalle particolarità dei singoli casi. Tale difficoltà potrebbe però essere ridotta dal fatto che ne1la ricostruzione della regola si rinvia a valori ${ }^{79}$ che sono coinvolti dai

limitato potrebbe sopravvivere se la gamma a cui deve mostrarsi adeguata fosse ampliata”: così Dworkin, R., op. cit., nota 13, pp. 230 e ss.

78 Ricoeur, P., "Conclusioni", Ars Interpretandi, n. 1, 1996, p. 91. Contrapposto al binomio differenza-disuguaglianza c'è quello della uguaglianza-identità, senza che, per questo, l'identità diventi un presupposto necessario dell'uguaglianza a scapito delle differenze. Una analisi puntuale delle teorie postmoderne che accoglie l'idea di una possibile "combinazione di pluralismo, tolleranza e rispetto per gli altri", piuttosto che quella di una giustizia che sia realmente in grado di comprendere i diritti fondamentali nella loro universalità. $C f r$. Rosenfeld, M., "Teorie della giustizia", Enciclopedia delle Scienze Sociali, vol. IX, 2001, p. 153.

79 Sul rapporto regola-principio-valore: "In astratto, si può dire che non c'è regola che non risponda a un principio e non c'è principio che non si colleghi ad un valore... Il che sta a dire che il nesso di congruenza tra valori-principi-regole è costitutivo della validità del diritto, è un assioma che precede addirittura il diritto costituzionale posto; è qualcosa che viene prima dello stesso potere di fare una costituzione. Questa può fare molte cose ma non disporre l'assurdo affermando contemporaneamente che una norma è arbitraria e ciò non di meno è valida...": così Zagrebelsky, G., "Diritto per: valori", 
casi concreti ma che non si esauriscono nei casi particolari e che, a loro volta, possono essere più o meno trincerati.

Se volessimo ridurre il discorso ai minimi termini, potremmo dire che se da un lato il precedente rappresenta il parametro di riferimento del passato proiettato nel futuro, ${ }^{80}$ il diritto vivente è il tertium comparationis idoneo ad indirizzare lo sguardo del giudice verso il futuro in un'ottica non solo di continuità con il passato,${ }^{81}$ di cui si rispetterebbe la matrice dei principi, ma anche di cambiamento, di orientamento de-

cit., nota 72, p. 877. Anche Angiolini, V., "Costituzione tollerante, costituzione totale ed interpretazione della disciplina delle libertà", La tutela dei diritti fondamentali davanti alla Corte Costituzionale, Torino, Giappichelli, 1994, pp. 31 e ss. Sul modo di intendere la giurisprudenza della Corte, per un verso si guarda all'attività di interpretazione in cui si sceglie un valore piuttosto un altro; per altro, si considerano le decisioni "come se" fossero tenute a rispettare le tradizionali tecniche interpretative che garantirebbero una certa "coerenza" ma non 1" "esattezza", la correttezza del risultato, Dogliani, M., "Il «posto» del diritto costituzionale", Giur. Cost., 1993, pp. 533 e ss. Sulla ricerca della regola per il caso concreto e l'uso del kantiano giudizio riflettente, Ricoeur, P., "Conclusioni", cit., nota 78, p. 196. Sulla distinzione tra norme e valori, per cui le prime si riferiscono ad un agire deontologico ed i secondi, ad un agire teleologico; sulle differenti conseguenze che produce l'applicazione della norma rispetto al valore; sui diritti fondamentali che intesi come norme "disciplinano una materia nell'eguale interesse di tutti; come valori essi configurano -insieme ad altri valori-un ordine simbolico esprimente identità e forma di vita di una particolare comunità giuridica", Habermas, J., Fatti e norme, Milano, Guerini e Associati, 1996, pp. 302 e ss. Sulla concezione universale di diritti umani che si scontra non con il relativismo degli stessi ma con il particolarismo (il riferimento è ai diritti delle minoranze), il pluralismo comprensivo propone un dialogo tra le norme di primo livello -relative alle diverse concezioni del bene- e quelle di secondo livello -che richiedono il riconoscimento della uguale dignità delle norme di primo livello-, Rosenfeld, M., "Can Human Rights Bridge the Gap Between Universalism and Cultural Relativism? A Pluralist Assessment Based on the Rights of Minorities", in Columbia Human Rights Law Review, 30, 1999, pp. 276 e ss.

80 Schauer, F., "Precedent", in Stanford Law Review, 39, 1987, p. 589: "If the future must treat what we do now as presumptively binding, then our current decision must judge not only what is best for now, but also how the current decision will affect the decision of other and future assimilable cases".

81 Sul compito del legislatore «costretto sempre più spesso a regolare i rapporti già trascorsi nel passato» e su quello della giurisprudenza di guardare al futuro attraverso la funzione del diritto vivente, si veda D'Amico, M., op. cit., nota 57, p. 56, e Zagrebelsky, G., "La dottrina del diritto vivente", Giur. Cost., 1986, I, pp. 1148 e ss. 
lle aspettative. ${ }^{82}$ La risoluzione delle controversie da parte dei giudici attraverso il precedente o il diritto vivente e dunque decidendo secondo determinati valori piuttosto che secondo altri, risponderebbe a mere esigenze di concretezza e di certezza del diritto, considerato che, anche il diritto vivente può costituire - qualora il giudice lo utilizzasse come alibi per trincerarsi e non come indice di prevedibilità- un modo per arroccarsi su una posizione evitando di non intervenire sul campo minato della interpretazione.

Nel contesto giuridico attuale, il rapporto dialettico tra i fatti e le norme trova nel diritto vivente un elemento in grado di focalizzare nella loro storicità le istanze che emergono nel contesto sociale. Infatti, con la dottrina del diritto vivente si recepisce e si consolida la interpretazione di disposizioni legislative secondo un orientamento stabile e diffuso delle magistrature superiori e che, pertanto, contribuisce a garantire una forma - seppur giurisprudenziale — di certezza del diritto. ${ }^{83}$ Dall'altro lato, invece, qualora il giudice utilizzasse il diritto vivente come tertium comparationis nelle sue decisioni, allora, favorirebbe delle alternative interpretative aprendo verso altre letture quei processi ormai consolidati da atti/leggi costituzionali.

\section{El diritto vivente}

Il contesto in cui l'uomo quotidianamente si pone è dato dall'esperienza, dagli atti e dai processi: i fatti possono essere percepiti come "esperienza meramente pratica" (Erfahrung) o, se rappresentativi

82 Sulla funzione simbolica delle "costituzioni normative"... che «oltre a servire da espressione simbolica della "coesistenza", "libertà", "uguaglianza”, "partecipazione” ecc... implicano... un orientamento delle aspettative di comportamento», si veda Neves, M., Costituzionalizzazione simbolica e decostituzionalizzazione di fatto, Lecce-Cavallino, Pensa, 2004, p. 31.

83 Sul punto, Anzon, A., "Il giudice a quo e la Corte costituzionale tra dottrina della interpretazione conforme a Costituzione e dottrina del diritto vivente", Giur. Cost., 1998, p. 1085 . 
di un quid ideale, di una "essenza", ${ }^{84}$ come "esperienza consapevole di vita" (Erlebnis). ${ }^{85}$

Nel processo dialettico tra la legge e la Costituzione il diritto vivente si inserisce esortando i giudici ad un'attività interpretativa dei principi tale per cui l'intentio legislatoris risulta essere in continuo divenire. Dal momento che il diritto vivente è il simbolo concreto della evoluzione degli orientamenti giurisprudenziali, esso è uno dei parametri cui la Corte guarda nel valutare la legittimità costituzionale della disposizione. È possibile, però, che si verifichi l'ipotesi in cui un nuovo diritto vivente si consolidi in un momento successivo all'atto introduttivo del giudizio incidentale, per cui, anche se consolidatosi in tempo successivo all'ordinanza di rimessione, è idoneo a privare di fondamento questioni di costituzionalità che si sono prodotte sulla base di interpretazioni e applicazioni contrarie al diritto vivente stesso. I problemi sorgono a

84 Parlare di "essenza" vuol dire avere «coscienza di qualcosa, di un oggetto su cui essa si dirige»; vuol dire distinguerla da una mera rappresentazione dei fatti, così Husserl, E., Idee per una fenomenologia pura e per una filosofia fenomenologica, Libro Primo, Torino, Einaudi, 1965, pp. 19 e ss. Su Husserl, E., v. Geymonat, L., op. cit., nota 9, pp. 18 e ss. Sull'essere la coscienza "intenzionale", perché sempre coscienza "di qualcosa", Husserl, E., "Postille alle Idee”, in op. cit., Libro Terzo, pp. 917 e 931: "L'elemento essenziale di novità... è la scoperta che una concreta descrizione della sfera coscienziale in quanto sfera chiusa dell'intenzionalità (perché solo così si dà concretamente) ha un senso completamente diverso da quello delle descrizioni della natura e quindi di quelle delle esemplari scienze naturali descrittive".

85 "Non basta affermare che ogni coscienza è coscienza-di e poi distinguere, quanto al tipo, i vari modi di coscienza... bisogna bensì interrogare le diverse categorie di «oggetti», puramente in quanto oggetti di una coscienza possibile, e risalire alle forme dell'essenza, che vanno sinteticamente collegate, delle «molteplicità» possibili, attraverso le quali la coscienza della identità di ogni singolo oggetto della categoria in questione perviene ad una sintesi descrivibile. Un unico e medesimo «oggetto simpliciter» percorre, in quanto intenzionalmente inteso, tutte queste molteplicità": così Husserl, E., op . cit., Libro Terzo, 932. Il concetto di «Erlebnis» è utilizzato da, Gadamer, H. G., Verità e metodo, Milano, Bompiani, pp. 91 e ss., il quale rinvia sia al relativismo storico di Dilthey per cui «il concetto di Erlebnis mediante la riflessività, l'interiorità, si propone... di giustificare gnoseologicamente la conoscenza del mondo storico. I dati primari a cui si rifà l'interpretazione degli oggetti storici non sono dati forniti dall'esperimento e dalla misurazione, ma unità significative»; sia a quella di Husserl per cui «il concetto di Erlebnis diventa il nome che si applica a tutti gli atti della coscienza, la cui essenza costitutiva è l'intenzionalità». La distinzione concettuale tra Erfahrung ed Erlebnis come innesto del fenomeno costituzionale è stata recentemente trattata da Carducci, M., Tecniche costituzionali, cit., nota 48, p. 53. 
questo punto per il fatto che sia il diritto vivente che il giudizio di rilevanza di una questione costituiscono due aspetti paralleli del concetto di "concretezza": il primo individua il modo in cui una disposizione è applicata nell'ordinamento e il secondo rileva con riferimento alla fattispecie esaminata dal giudice a quo. Inoltre, se il diritto vivente incide indubbiamente sull'operato della Corte Costituzionale, non così per il giudice comune, che sarebbe garantito comunque dall'art. 101 Cost.: sono separati i piani dell' interpretazione della legge per valutarne la sua applicabilità (sul quale il diritto vivente agisce in forza di precedente), da quello di interpretazione della legge al fine di valutarne la sua costituzionalità. Sul piano processuale, il giudice $a$ quo è libero di disattendere quanto proposto dalla norma vigente; non può dare applicazione alla norma contenuta nell'ordinanza di rimessione; né può ripresentare la identica questione di rilevanza alla Corte, con eventuale integrazione dell'ordinanza. La Corte, d'altra parte, non potrebbe imputargli il fatto di non essersi adeguato ad un orientamento stabile, stando la libertà del giudice di rifiutare il diritto vivente.

$\mathrm{Nel}$ caso in cui un diritto vivente non si sia ancora formato in merito a leggi o questioni sulle quali la Corte sia chiamata a pronunciarsi, essa potrà ricorrere allo strumento delle sentenze interpretative che operano su un piano sostanzialmente astratto rispetto a quello concreto del diritto vivente. Il passo successivo per valutare il comportamento della Corte in termini di tempestività sarà quello di guardare alla iniziativa del giudice $a$ quo e non all'approvazione dell'atto legislativo, per cui il diritto vivente potrebbe formarsi nel periodo che intercorre tra l'entrata in vigore di una legge ed il giudizio incidentale - avendo tale dottrina "la pretesa di fissare le coordinate del sindacato di costituzionalità non solo per l'ipotesi di esistenza di un indirizzo interpretativo- applicativo uniforme e consolidato, ma anche per il caso opposto di un diritto vivente assente o tutt'ora in fieri'. ${ }^{86} \mathrm{Il}$ sindacato della Corte, inciderebbe su un elemento concreto per valutarne la legittimità oppure contribuirebbe a formare una consuetudine giurisprudenziale ${ }^{87} \mathrm{o}$ amministrativa orientandola secondo una interpretazione conforme a Costituzione.

86 Pugiotto, A., op. cit., nota 76, p. 632.

87 Nell'ambito del mutamento tacito della Costituzione per «carente applicazione, in modo uniforme e costante, di una norma posta dalla Costituzione», si veda Q. Camerlengo, op. cit., nota 47, pp. 256 e ss. 
Il diritto vivente (anche nella sua variante regolamentare) ${ }^{88}$ può ritenersi un "dato del giudizio costituzionale" nonché - qualora si fosse già formato - l'elemento principale oggetto dell'attività della Corte tesa a non creare "zone franche" sconosciute alla prassi e ad utilizzare invece come tertium comparationis un orientamento consolidato nella prassi giurisprudenziale (e amministrativa) con cui far emergere le istanze della società. ${ }^{89} \mathrm{Si}$ può dire, perciò, che

non esiste alcuna sostanziale garanzia, in base alla quale le leggi che pretendono di basarsi sulla Costituzione non la contravvengano nonostante tutto, causando così un mutamento costituzionale non voluto o, per lo meno, non esplicitamente perseguito. I precetti costituzionali spesso sono oscuri ed estesi e solo il legislatore conferisce loro un significato preciso, attraverso leggi che li rendano concreti in modo molto simile a ciò che fa il giudice, quando assume chiara coscienza del contenuto delle disposizioni da applicare. ${ }^{90}$

Considerando il passato ed il futuro quali orizzonti di possibilità, per cui la realtà non sarebbe altro che la differenza tra ciò che è attuale e ciò che è potenziale, l'attribuzione di senso consente di rendere presente il passato. Sul piano giuridico, ad esempio, con riferimento ai fatti che sono "cristallizzati" nel precedente (e che rappresenta il tempo passato), il diritto vivente identifica un preciso contesto culturale colto in un tempo presente (non fosse altro che per la sua accezione di diritto "vivente") ma supportato dall'elemento del precedente in grado di concretizzarlo. Il diritto è vivente in quanto ritematizza il passato attribuendo un senso ad un determinato "bene"; la concezione di questo "bene", si apre ad altre possibilità interpretative nel caso in cui è oggetto di una ulteriore attribuzione di senso, cioè di un rinvio da parte del giudice.

Nel momento in cui il giudice interpreterà tali fatti, dovrà supportare la scelta con alcuni valori piuttosto che con altri, con "argomenti di principio ricavati da diritti costituzionali o dal diritto to equal concern che dei primi è la matrice". ${ }^{91}$

88 Bruno, A., op. cit., nota 19, pp. 454 e ss.

89 Pugiotto, A., op. cit., nota 76, pp. 641-651.

90 Jellinek, G., op. cit., nota 51, p. 23. Ainis, M., Le parole e il tempo della legge, Torino, Giappichelli, 1996, pp. 42 e ss.

91 Chessa, O., "Corte Costituzionale e trasformazioni della democrazia pluralistica", in Tondi della Mura, V. et all. (cur.), op .cit., nota 19, p. 65 (nt 128); Dworkin, R., op. cit., nota 13, p. 229. 
Davanti ai c.d. hard cases che richiedono scelte difficili la dimensione temporale, in questi specifici casi più che in altri, è un elemento che consente di cogliere la trasformazione sociale per percepirne i cambiamenti, per individuare quei fatti che rompono con la tradizione, che si presentano nuovi e orientati verso una dimensione futura, portatori di un'aspettativa di un singolo o di un gruppo. Alla ricerca di una soluzione delle controversie, si assiste ad un processo di "svuotamento" della portata dei principi suddetti dal momento che raramente la Costituzione si impone come norma direttamente obbligante e, dunque, i suoi principi come diretta applicazione al caso concreto. Vi sono decisioni, ${ }^{92}$ in cui la norma costituzionale costituisce un principio generale già presente nell'ordinamento (art. 12 disposizioni preliminari Codice civile italiano) ed altre in cui la norma costituzionale concorre con la legge nella valutazione di un fatto. Si tratta di casi in cui l'attività interpretativa del testo fa leva sui principi costituzionali alla stregua di mero argomento, pari a qualsiasi altro ed in cui il ruolo della legge introduce, assicura e disciplina i principi della Costituzione - come se il giudice volesse rafforzare il contenuto di una norma ordinaria ancorandola ai principi costituzionali, senza una vera e propria applicazione diretta della Costituzione-. Questa tecnica di risoluzione della controversia — per cui la Costituzione entra a far parte del momento decisorio solo attraverso il rinvio ad essa fatto dalla legge - sembra riservare alle norme costituzionali un ruolo non solo secondario ma anche scisso, in qualche modo, dall'ordinamento, per cui i principi costituzionali sarebbero richiamati solo al fine di valutare la conformità ad essi della legge e la sua applicabilità alla fattispecie. ${ }^{93}$ Il dettato costituzionale risulta ancora una volta indebolito nel momento

92 Ad. Plen., n. 20/92, in Cons. St., 1992, I, 1765.

93 Sul testo costituzionale quale limite (e non fondamento) alla creatività dell'interprete, Pace, A., "Metodi interpretativi e costituzionalismo", Quaderni Costituzionali, 2001, n. 1, p. 45. Sulla possibilità per i giudici di creare diritto, Guastini, R., op. cit., nota 54, pp. 11-12. Attraverso una funzione "normogenetica" i principi della Costituzione esprimono l'aspetto prescrittivi di un sistema giuridico, mentre secondo una funzione "sistemica" l'accento sarebbe posto sull'attività dell'interprete che dovrà valutare il significato di una norma a confronto con l'intero ordinamento e di «sceverare e porre in evidenza» l'identità stessa dell'ordinamento. Magri, M., La legalità costituzionale dell' Amministrazione, Milano, Giuffrè, 2002, p. 225. Con riferimento al problema relativo all'uso del principio, cioè alla scelta "fra l'utilizzazione del «principio» in funzione «integratrice» oppure l'utilizzazione della «norma precisa» (re)interpretata per rapporto al «principio» medesimo", si veda Angiolini, V., op. cit., nota 80, pp. 37 e ss. 
determinante della concretizzazione dei principi e della tutela delle istanze dei cittadini. La stessa legge chiamata a specificare i principi costituzionali potrebbe richiedere in futuro ulteriori interventi normativi per poter essere applicata o, semplicemente, potrebbe riservare la disciplina di una materia ad un regolamento successivo; potrebbe necessitare di una attività di attuazione che, quale attività normativa rinvia, per completarsi, ai principi sottesi alla lettera della legge (procedimento che ritroviamo anche nelle additive di principio). "Quanto più lacunoso e generico è il dettato della Costituzione, tanto più si eleva la possibilità che essa in futuro sia "aggredita" da atti normativi sottostanti». ${ }^{94}$

\section{Non deference e judicial minimalism}

L'autorevolezza delle decisioni costituzionali della Corte Suprema degli Stati Uniti è stata determinante nella sentenza Brown v. Board of Education. ${ }^{95}$ In quell'occasione, il giudice E. Warren ha dichiarato l'annullamento di quanto stabilito nel precedente caso Plessy $v$. Ferguson, ${ }^{96}$ rifiutando il principio "separato ma eguale" fino ad allora ritenuto legittimo e basilare nel processo di segregazione razziale.

La sentenza Brown v. Board of Education fu profondamente dibattuta dal momento che la clausola della equal protection, in nome della quale si era pronunciato il giudice Warren, così come ratificata dai Padri Fondatori, non diceva e non dice nulla con specifico riferimento alla segregazione razziale. "A volte, come in Brown v. Board of Education, è essenziale che la Corte parli con una sola voce", ${ }^{97}$ perciò, l'autorevolezza della fonte risolse il caso con una sentenza che guardava ai cambiamenti sociali, adeguandosi ad essi per quanto possibile ed avviando il lungo e travagliato processo di integrazione razziale. L'atteggiamento di coloro i quali disapprovarono la decisione del 1954 chiama alla mente quella particolare corrente di pensiero nota nel dibattito statunitense come nonDeference che si verifica "allorquando un funzionario non giudiziario dissente su una questione giudiziale riguardo ad una questione costi-

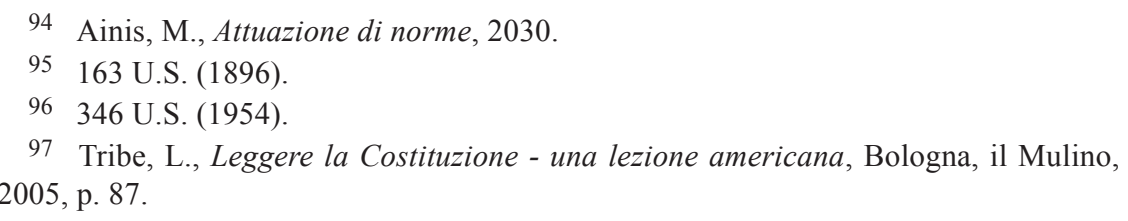


tuzionale e non adegui i propri atti a quella decisione, arrivando persino a contrastarla apertamente". ${ }^{98}$

Si tratta di una posizione che trova una sua giustificazione in tre motivi. Secondo il primo, la non-Deference risponde, da un lato, all'esigenza di non sentirsi vincolati a decisioni imposte dagli organi giudiziari nell'esercizio del loro potere; dall'altro, ad una strategia politica secondo la quale è considerata poco rilevante l'eventuale incompatibilità, "nonadeguatezza" a precedenti consolidati in assenza di disincentivi politici o legali (Real politik). Per il secondo motivo, non si tratterebbe di una particolare forma di non-Deference dei funzionari esecutivi e legislativi ma, semplicemente, di una mancanza di competenza di tali organi (per il principio della separazione dei poteri) in ambito di interpretazione costituzionale. Infatti, se le decisioni concernenti i diritti rientrano nella competenza delle Corti, quelle politiche (non necessariamente in senso stretto ma per lo più tese alla massimizzazione del bene pubblico) spettano al potere legislativo. È evidente la connessione con il primo profilo se si considera che talvolta il Congresso si adegua a precise opinioni di interpretazione costituzionale con lo scopo di perseguire determinati risultati politici. Infine, la non-Deference rappresenta una vera e propria forma di "disobbedienza" dei funzionari non giudiziari, nel significato più concreto del termine, cioè come di mancata accettazione di una decisione altrui, ritenuta vincolante ma non condivisa nel contenuto. ${ }^{99} \mathrm{Il}$ problema che emerge, dunque, è un problema di fedeltà costituzionale che, nella prospettiva della non-Deference implica una consequenziale infedeltà alle decisioni costituzionali della Corte qualora queste non siano condivisibili.

Dal momento che la mera funzione risolutiva di una decisione non è sufficiente perché una fonte sia considerata autorevole, è necessario rintracciare le condizioni in grado di soddisfare il requisito di autorevolezza. Tali condizioni si rinvengono nelle esigenze di stabilità e di coordinamento, ritenute le funzioni principali di una Costituzione chiamata, nel tempo, ad una continua tutela del bene comune. Affinché esse possano

98 Schauer, F. e Alexander, L., L'interpretazione stragiudiziale della Costituzione, Lecce-Cavallino, Pensa, 2006, p. 27.

99 È una forma di non Deference, che richiede l'obbligo da parte dei funzionari di obbedire alla Costituzione, «ancorché non ammette che i funzionari non giudiziali abbiano l'obbligo di obbedire alle Corti nel determinare come obbedire alla Costituzione», Schauer, F. e Alexander, L., op. cit., nota 98, p. 34. 
realizzarsi pienamente, devono trovare nella interpretazione costituzionale fornita dalla Corte Suprema, fonte autorevole e sovrana per eccellenza, una loro concretizzazione. "Un buon disegno istituzionale richiede norme che inducano coloro che prendono decisioni a rimettersi alle decisioni di altri con cui non sono d'accordo. Alcuni denominano questo assunto positivismo. Altri lo chiamano formalismo. Noi lo chiamiamo Law". 100

Il contesto statunitense costituisce terreno fertile per il sorgere di un altro particolare atteggiamento di cui si fa portatore non più il funzionario amministrativo, legislativo o esecutivo ma il giudice stesso. Infatti, in virtù di esigenze di real politik, talvolta i giudici decidono esprimendo il loro giudizio tanto quanto basta per giustificare il risultato cui pervengono; lasciando per quanto possibile le cose undecided alimentano quel fenomeno conosciuto come judicial minimalism, ${ }^{101}$ di cui l'analogia costituisce lo strumento tipico. Le soluzioni così adottate restano aperte e offrono una possibilità al processo democratico di intervenire nella formazione di ulteriori sviluppi delle soluzioni. Il judicial minimalism tende a caldeggiare la necessità sia di una giustificazione adeguata al caso concreto sia di decisioni fondamentali prese da soggetti democraticamente responsabili, rivelandosi determinante nel caso di questioni particolarmente complesse, profondamente sentite dalla società e che possono in qualche modo essere un segnale, un indice di mutamento di sensibilità nei confronti di tematiche profonde, che operano su larga scala. Qualora le decisioni, in questi casi, fossero prese attraverso formule "chiuse", immobili, nel senso di immodificabili, si correrebbe il rischio di una soluzione inefficiente in quel determinato momento storico o, sebbene giusta, probabilmente scorretta in un tempo futuro: "Facts and values can go in unanticipated directions, thus rendering anachronistic a rule that is well-suited to present conditions". ${ }^{102}$

Uno dei fattori che maggiormente induce a pensare che in determinati casi il Minimalismo sia il metodo più adeguato per raggiungere risultati corretti è dato dalla carenza, in uno specifico settore, di informazioni: si

100 Ibidem, p. 93.

101 Sunstein, C. R., "Foreword: Leaving things undecided", in Harvard Law Review, 110, 1996, pp. 4-101.

102 Ibidem, p. 17; Stone-Seidman-Sunstein-Tushnet-Karlan, Constitutional Law, New York, Aspen, 2005, pp. 678-679. 
pensi, ad esempio, al caso in cui "in un tempo, potremmo pensare che le relazioni omosessuali siano simili ad un incesto; in un altro, potremmo considerare bizzarra tale analogia". ${ }^{103}$ Il tema relativo alla possibilità per gli omosessuali di contrarre matrimonio o di adottare, come quello della eutanasia o della fecondazione eterologa, sollevano questioni legate non solo al ruolo dell'individuo nel contesto in cui agisce ma soprattutto problematiche che coinvolgono il bilanciamento di interessi, il criterio di ragionevolezza (intesa come "congruità degli atti giuridici con la sfera del fatto, degli interessi" e come "bilanciato rapporto tra i diversi principi coinvolti dalle diverse fattispecie, dai diversi casi giuridicamente rilevanti"), ${ }^{104}$ di proporzionalità e coerenza dell'intero sistema. ${ }^{105} \mathrm{Se}$ le tematiche in esame non sono sollevate oppure se sollevate sono risolte negativamente, probabilmente ciò si verifica perché quella determinata comunità avverte la necessità della prudenza nel sostenere certe rivendicazioni all'interno di un sistema, di un processo democratico che non vuole o non è ancora culturalmente preparato ad accoglierle. "Constitutional law is a peculiar mixture of substantive theory and institutional constraint"106 per cui, sebbene in un determinato periodo storico possano verificarsi fatti e cambiamenti di circostanze, magari supportati da manifestazioni sociali, popolari (si pensi al referendum sulla fecondazione eterologa, ai dibattiti recenti sull'eutanasia e sulle coppie di fatto), essi comunque si muovono nell' «arena democratica». ${ }^{107}$ Questo comporta

103 Sunstein, C. R., Intese non completamente teorizzate e usi costruttivi del silenzio, Lecce-Cavallino, Pensa, 2004, p. 40.

104 D'Andrea, L., op. cit., nota 34, pp. 282-283.

105 Sulla distinzione tra ragionevolezza e razionalità, ossia tra adeguatezza ad un valore di giustizia e coerenza logica, si v. M. Cavino, Interpretazione discorsiva del diritto, Milano, Giuffrè, 2004, pp. 176 e ss., Carducci, M., Tecniche Costituzionali, cit., nota 48, pp. 79 e ss. Un'applicazione pratica e concreta dei problemi sollevati dal bilanciamento si rinvengono nella sentenza della Corte Cost. del 27 ottobre 1988 n. 992 in Angiolini, V., op. cit., nota 80, pp. 23 e ss.

106 Sunstein, C. R., "Foreword", cit., nota 101, p. 97.

107 Ibidem, nota 98. La rule of law come presupposto per realizzare una democrazia legittimata dal consenso in una società pluralista, partendo dall'analisi del Rechtsstaat tedesco, continuando con l'Etat de droit, è trattata da Rosenfeld, M., "The Rule of Law and the Legitimacy of Constitutional Democracy", Southern California Law Review, 74, 2001, p. 1348 e ss. La democrazia è stata, di recente, autorevolmente identificata attraverso dieci punti fondamentali: La fede in qualcosa che vale; la cura delle personalità individuali; lo spirito del dialogo; lo spirito della uguaglianza; l'apertura verso chi è portatore di 
una esigenza di compatibilità tra i nuovi diritti che spingono per imporsi ed il sistema costituzionale di riferimento che dovrebbe comprenderli.

Il Minimalismo (e, quindi, il rinvio all'analogia ed al precedente) può, talvolta, sembrare la soluzione più ovvia ma anche la più sicura a tutela dell'equilibrio interno di un sistema democratico: i giudici evitano le grosse questioni costituzionali; preferiscono ricorrere all'uso del precedente anche per limitare le occasioni per un intervento giudiziale in questioni politiche; cercano di ridurre i rischi di errore che teorie astratte potrebbero invece introdurre e di ampliare lo spazio per le decisioni democratiche; sembra, infine, una soluzione al problema del raggiungimento di un consenso all'interno del pluralismo attraverso astrazioni non completamente specificate e non completamente teorizzate: "Sometimes the best way for the Court... is by leaving things undecided". ${ }^{108}$

Vi sono alcune Costituzioni — come quelle dell'Europa dell'Est e del Sud Africa - che sono sorte sulla base di norme astratte non del tutto specificate, sulla base di accordi non completamente teorizzati che trovano un loro riferimento al caso particolare nel momento della loro applicazione. Questo significa che, pur avendo chiaro il proprio ruolo all'interno di un contesto, ${ }^{109} \mathrm{i}$ cittadini (e spesso anche $\mathrm{i}$ giudici) riescono a formare un accordo su principi di medio livello ma non a livello più elevato, più teorico né sui casi particolari; ancora, sui risultati da conseguire ma non completamente a livello teorico, per cui, quel determinato risultato verrebbe supportato esclusivamente da principi di basso livello, cioè caratterizzati da una portata piuttosto relativa, limitata. Così, spesso ci si trova d'accordo nello stabilire un rapporto analogico, di similitudine tra i casi in esame senza però comprendere, in parte o totalmente, la teoria generale che ne è il fondamento. In questo senso, il processo analogico tende a dare una soluzione immediata al caso in esame, una soluzione

identità diverse; la diffidenza verso decisioni irrimediabili; l'atteggiamento sperimentale; la coscienza della dignità dell'essere maggioranza e minoranza; l'atteggiamento altruistico; la cura nell'uso delle parole: cfr. Zagrebelsky, G., su La Repubblica del 04.03.2005.

108 Sunstein, C. R., "Foreword”, cit., nota 101, pp. 99-101.

109 Sul tema degli “attributi strutturali”, quali il genere, l'età, lo status familiare... quasi sempre stabiliti dalla legge e degli "attributi sociali" come burocrate, omosessuale, senzatetto... strettamente connessi alle aspettative per cui le «pratiche di individualizzazione obbediscono ad un interesse di distinzione», mentre quelle di «classificazione obbediscono a un interesse di controllo», Sparti, D., Soggetti al tempo, Milano, Feltrine1li, 1996, pp. 141 e ss. 
fondata sulla ricerca di principi tra loro non incompatibili ${ }^{110}$ sebbene tesi alla realizzazione di una "coerenza locale e parziale»" ${ }^{111}$ dal momento che essi vanterebbero una portata piuttosto relativa, di basso livello. Inoltre, il procedimento analogico non può essere definito a priori conservativo, richiedendo, invece, una valutazione caso per caso tale per cui solo la natura dei principi richiamati nella decisione potranno qualificarla come "trincerata" o meno; difficilmente, però, rinviando al precedente ed adottando il metodo analogico il giudice perverrà alla costruzione di teorie e alla elaborazione di principi nuovi. Ad ogni modo, si riesce ad arrivare alla soluzione pur non essendoci accordo sui principi che ne sono il fondamento, e questo perché l'obiettivo è quello di "rendere possibile l'accordo dove l'accordo è necessario e rendere non necessario l'accordo dove esso è impossibile". ${ }^{112}$ Non ci sarebbe in questa operazione la pretesa di fermare il tempo: infatti, la possibilità di una soluzione della controversia potrebbe essere determinata dall'esistenza di un accordo sulla portata, sul valore di una norma o sul ricorso ad una analogia valida e non vi potrà mai essere la certezza che questo porti ad una decisione equa, bilanciata, giusta perché è possibile che nel ragionamento analogico si incorra in fraintendimenti, differenze o somiglianze tra casi a confronto che potrebbero non essere interpretate correttamente.

I problemi con le analogie e i livelli mediocri di pensare, dovrebbero indurci ad essere più ambiziosi. Potremmo essere sospinti nella direzione della teoria generale - e verso diritti più ampi e forse più controversi- precisamente perché i ragionamenti analogici forniscono una ragione inadeguata e non completamente teorizzata di rilevante somiglianza o di rilevante diversità. Tutto ciò dovrebbe essere sufficiente per dimostrare che il valore delle decisioni basate su norme o su analogie sono parziali. ${ }^{113}$

Potrebbe emergere 1'utilità di tali "accordi non completamente teorizzati" in quanto essi consentono di decidere le controversie in tempi brevi limitando lungaggini processuali e costi di conflitti duraturi, favorendo una certa stabilità per l'intero sistema; garantiscono risparmio di tempo e rintracciano soluzioni possibili — il precedente, ad esempio, "libera i

110 Sunstein, C. R., Intese, cit., nota 103, pp. 47-48.

111 Ibidem, p. 50.

112 Ibidem, p. 26.

113 Ibidem, p. 30. 
giudici" dal prendersi cura di un certo numero di casi; in quei sistemi in cui esso (il precedente) è un dato imprescindibile, costituiscono ovviamente dei pilastri fondamentali e rappresentano un elemento prezioso nello sviluppo di concetti nel tempo- si consideri quello di uguaglianza o quello di libertà. Nell'utilizzazione degli strumenti a sua disposizione, il giudice deve tendere a colmare la frattura tra la legge ed il diritto. Ogni soluzione del caso concreto non potrà però mai dirsi esaustiva perché $\mathrm{i}$ valori costituzionali non potranno mai essere pienamente realizzati ed ogni sentenza potrà ritenersi falsificabile da quella successiva. Quanto detto è facilmente immaginabile se si considera che, nel lungo periodo, innumerevoli sono le incognite che sfuggono alle tecniche di bilanciamento e, tuttavia, il giudice è chiamato a pronunciarsi, indipendentemente che si tratti di hard or easy cases: ${ }^{114}$

la storia, dialettica della durata, non è forse a suo modo spiegazione della realtà sociale in tutta la sua verità? E quindi spiegazione dell'attualità?...non bisogna pensare solo in termini di tempo breve, credere che solo i personaggi che fanno del rumore siano i più autentici. Ve ne sono altri silenziosi...ma chi non lo sapeva già? 115

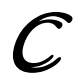

114 Schauer, F., "Easy cases", Southern California Law Review, 58, 1985, p. 399.

115 Braudel, F., "Storia e scienze sociali. La «lunga durata»", La storia e le altre scienze sociali, Bari, Laterza, 1974, p. 172.

Fecha de recepción: 24 de marzo de 2010.

Fecha de dictamen: 7 de mayo de 2010. 